\title{
Mechanisms of Molecular Ferroelectrics Made Simple
}

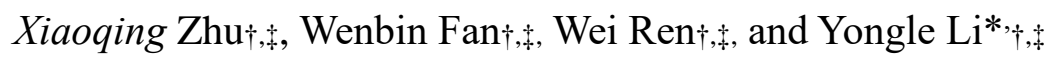

$\dagger$ Department of Physics, Shanghai University, Shanghai 200444, China.

¥ Department of Physics, International Center of Quantum and Molecular Structures, Shanghai University, Shanghai 200444, China.

*Email: yongleli@shu.edu.cn; 
Molecular ferroelectrics have captured immense attention due to their superiority over inorganic oxide ferroelectrics, such as environmentally friendliness,, low cost, flexibility, and foldability. However, the mechanisms of ferroelectric switching and phase transition for the molecular ferroelectrics are still missing, which leads to the development of less efficient novel molecular ferroelectrics. In this work, we have provided a protocol by combining molecular dynamics simulation on a polarized force field named polarized crystal charge and an enhanced sampling technique, replica-exchange molecular dynamics, to simulate such mechanisms. With this procedure, we have investigated a promising molecular ferroelectric material, $(R) /(S)$-3-quinuclidinol crystal. We have simulated the ferroelectric hysteresis loops of both enantiomers and obtained spontaneous polarization as $7.4 \pm 0.1$ and $7.8 \pm 0.1$ $\mu \mathrm{C} \cdot \mathrm{cm}^{-2}$, respectively, and the corresponding coercive electric field as $15 \mathrm{kV} \cdot \mathrm{cm}^{-1}$. We have also found the Curie temperature $\left(T_{\mathrm{c}}\right)$ to be $380 / 385 \mathrm{~K}$ for ferro-/paraelectric phase transition of both enantiomers. All of the simulated results are highly compatible with experimental values. In addition to this, we predict a novel $T_{\mathrm{c}}$ of about $600 \mathrm{~K}$. This finding is further validated by principal component analysis. Our work would promote the future exploration of multifunctional molecular ferroelectrics for the next generation of intelligent devices.
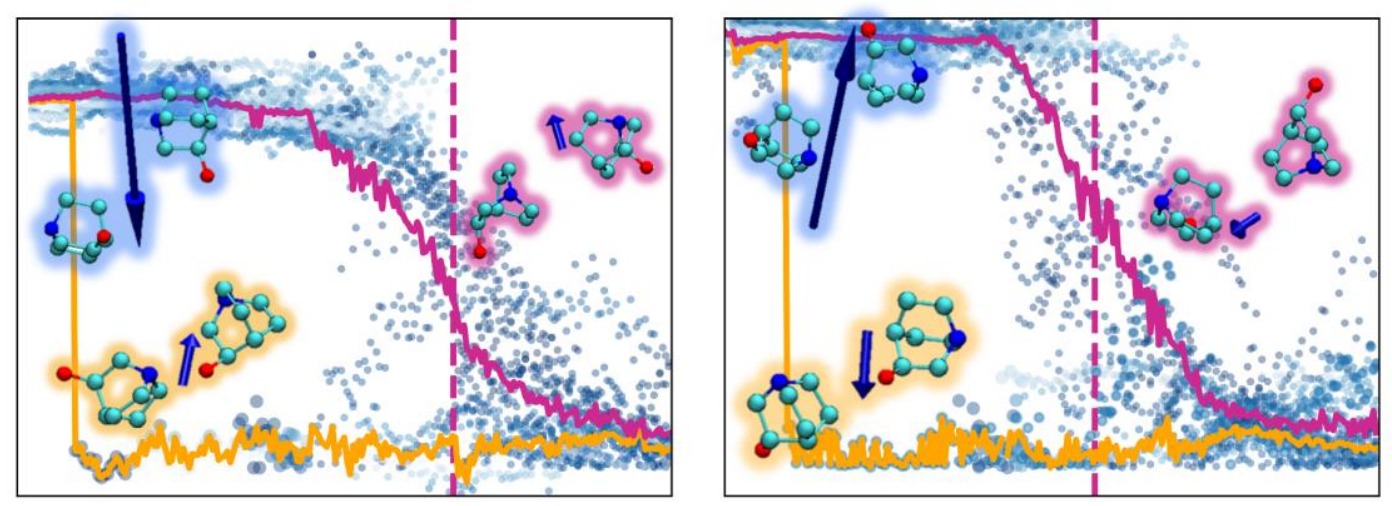


\section{Introduction}

As a functional material in electronic technology, ferroelectric materials have the characteristics such as non-volatile ferroelectric switching effects, piezoelectricity, pyroelectricity and nonlinear optical effects, and can be used to make non-volatile ferroelectric random access memory (FeRAM $)^{1}$, miniature piezoelectric drive motors, pyroelectric detectors, light modulators, and myriads of multifunctional devices ${ }^{2,3}$, which are widely used in high-tech industries such as electronic communications, biomedicine, national defense technology, and aerospace. ${ }^{4}$ Since the first ferroelectrics, Rochelle salt $\left(\mathrm{KNaC}_{4} \mathrm{H}_{4} \mathrm{O}_{6} \cdot 4 \mathrm{H}_{2} \mathrm{O}\right)$, discovered in $1920,{ }^{5}$ ferroelectricity is under extensive investigation until now. In the course of the development of ferroelectrics in the past century, a large number of different types of ferroelectric materials have been found, including inorganic oxides,,${ }^{6,7}$ molecular crystals, ${ }^{8}$ liquid crystals, ${ }^{9}$ polymers, ${ }^{10}$ etc. Traditionally, the most widely applied ferroelectrics in the industry are inorganic ceramics, such as $\mathrm{BaTiO}_{3}, \mathrm{~Pb}(\mathrm{Zr}, \mathrm{Ti}) \mathrm{O}_{3}(\mathrm{PZT})$, and $\mathrm{Pb}\left(\mathrm{Mg}_{1 / 3} \mathrm{Nb}_{2 / 3}\right) \mathrm{O}_{3}-\mathrm{PbTiO}_{3}$ (PMN-PT), or ferroelectric polymers such as polyvinylidene fluoride (PVDF). However, due to the preparation process, inorganic ferroelectrics have disadvantages of high toxicity, high sintering temperature, difficulty in preparation, and high cost, which limit their wider application. ${ }^{11}$ On the other hand, ferroelectric polymers are soft and flexible and can be easily customized to meet the requirements of next-generation flexible and wearable devices, though such polymers still have disadvantages, such as large coercive field $\left(E_{\mathrm{c}}\right)$ and uniaxial characteristicand their application is also limited.

In the recent two decades, organic molecular ferroelectric materials have become a good alternative to next-generation ferroelectrics for industrial usage, because of their multi-facet advantages, such as they are environmentally friendly, non-toxic, low cost, flexible and wearable. ${ }^{12}$ Molecular ferroelectrics not only have the properties of high Curie temperature $\left(T_{\mathrm{c}}\right)$ and high saturation polarization as large as those of inorganic ferroelectrics but also have the advantages of flexibility, structural tunability, and versatility, allowing them to be utilized in a myriad of cases. ${ }^{13,14}$ 
In 2019, a pair of molecular ferroelectric with chirality and high $T_{\text {c }}$, $(R) /(S)$-3-quinuclidinol, was prepared, ${ }^{15}$ which is highly promising for industrial usage, possessing large saturation polarization $\left(P_{\mathrm{s}}\right)$, relatively small $E_{\mathrm{c}}$, and high $T_{\mathrm{c}}$. Before this, the ferroelectrics containing a single chiral molecule were limited to chiral tartaric acid and with less value for industrial applications. 16 $(R) /(S)$-3-quinuclidinol is found in chemistry and medicine applications, and its ferroelectricity was predicted by molecular design. ${ }^{12}$ This is a breakthrough in the discovery of molecular ferroelectrics since other candidates proposed before suffer from low $T_{\mathrm{c}} \cdot{ }^{14}$

In addition to the triumphs of preparing molecular ferroelectric crystals in the study of molecular ferroelectrics, the mechanisms of the origin of spontaneous polarization, ferroelectric switching, and phase transition are still vague, and there is a lack of systematical investigations on such topic. Due to the missing of a thorough understanding of such mechanisms, the development of novel molecular ferroelectrics is still highly dependent on experience. ${ }^{17}$ Even worse, these mechanisms also cannot all be obtained from first principles calculations. However, the confirmation of such mechanisms is a key question that needs to be solved in the investigation of molecular ferroelectrics. ${ }^{18}$ So far, the research on molecular ferroelectricity is still far from enough, and a protocol for systematical investigation on such a topic is urgent.

Recently, our group proposed a protocol of molecular dynamics (MD) simulation for a kind of molecular ferroelectrics, the bio-ferroelectrics. ${ }^{19}$ It can give not only reasonable predictions of $P_{\mathrm{s}}, E_{\mathrm{c}}$, and $T_{\mathrm{c}}$, but also mechanisms of both ferroelectric switching and ferro-/paraelectric phase transition. This is an important basis for the study of the ferroelectric properties of materials.

For better understanding and making practical usages of the molecular ferroelectrics, and also validating our protocol, this work will study the $(R) /(S)$-3-quinuclidinol on the atomic scale, using the MD simulation methods with polarized crystal charge (PCC) to systematically elucidate the dynamics of ferroelectric switching under an external electrostatic field and phase transition with heating to obtain the mechanisms for both ferroelectric switching and 
ferro-/paraelectric phase transition processes and shed a light on the de novo design of such kind of molecular ferroelectrics in the future. The work in this paper can not only provide a reliable protocol for future theoretical and simulation work but also verify and guide the design of molecular crystal ferroelectric materials to promote the application of organic chiral molecular crystal ferroelectric materials in production and life.

\section{Computational Details}

The original molecular structures were obtained from the CCDC database, ${ }^{20}$ with entry ID $1869376(R)$ - and $1869377(S)$-: $(R)$-3-quinuclidinol [ferroelectric (paraelectric) space group: $\left[P 6_{1}\left(P 6_{12} 2\right)\right]$ and $(S)$-3-quinuclidinol $\left[P 6_{5}\left(P 6_{5} 22\right)\right]$. We optimized the crystal structure using the first principles calculations. As reported by experimental work, the single molecule of the chiral structure of $(R) /(S)$-3-quinuclidinol is in point group $6\left(\mathrm{C}_{6}\right) .{ }^{15}$ The first principles calculations were performed by the Vienna $A b$ initio Simulation Package (VASP) ${ }^{21}$ based on the projector augmented wave pseudopotentials. ${ }^{22}$ We used two exchange-correlation functionals, the pure generalized gradient approximation functional Perdew Burke Ernzerhof, ${ }^{23}$ and hybrid functional Heyd-Scuseria-Ernzerhof (HSE). ${ }^{24}$ The former is the most widely adopted, and the latter can give the accurate geometry and band gap for molecular crystals. Plane wave basis sets were used and the cutoff energy was set as $400 \mathrm{eV}$. The Brillouin zone was sampled with $5 \times 5 \times 1$ k-point in a Monkhorst-Pack grid. To optimize the structure, both the lattice constants and positions of all atoms are relaxed until the force is less than $0.01 \mathrm{eV} \AA^{-1}$ for all calculations, and the criterion for the total energy was set to be $10^{-6} \mathrm{eV}$. The VESTA package $^{25}$ was used to visualize crystal structures. Various functionals were carried out to optimize the structure of $(R)$-3-quinuclidinol and $(S)$-3-quinuclidinol, which shows that the crystal structure calculated by the HSE functional is closer to the experimental structure compared with other functionals, while the PBE functional underestimates the band gap by about $1-2 \mathrm{eV}$, therefore, the HSE functional is adopted in all following calculations. 
Especially, to deal with the periodic crystal model correctly, the $\mathrm{PCC}^{19,26}$ was incorporated into the force field recently developed by our group. In our previous work, we found that the $P_{\mathrm{s}}$ of $\gamma$-glycine simulated by PCC MD was about $53 \mu \mathrm{C} \cdot \mathrm{cm}^{-2}$, comparing with the first principles calculation result $70.9 \mu \mathrm{C} \cdot \mathrm{cm}^{-2}$, with a relative error of $25 \% .{ }^{19}$ In our another work on the polarization of DIPAC, DIPAB and DIPAI, the $P_{\mathrm{s}}$ from PCC MD was $5.4 \pm 0.3,5.0 \pm 0.4$ and $4.0 \pm 0.4 \mu \mathrm{C} \cdot \mathrm{cm}^{-2}$ respectively, and the corresponding results from first-principles calculation is $6.8,6.2$ and $5.3 \mu \mathrm{C} \cdot \mathrm{cm}^{-2}$. The relative error is 21,19 and $25 \% .{ }^{26}$ Therefore, we can conclude that our PCCcan give reliable spontaneously with moderate accuracy. Figures S3 and S4 and Tables S1 and S2 show the convergence of PCC values for all atoms. The Visual Molecular Dynamics (VMD) ${ }^{27}$ package is used for visualization.

The MD simulations at different temperatures and under external electric fields were carried out in the NAMD software package. ${ }^{28,29}$ To study polarization switching, we ran several $2 \mathrm{~ns}$ independent trajectories with different external electric fields as shown in Figure 2 at $310 \mathrm{~K}$ in the NVT ensemble. In order to reduce the size problem, a $6 \times 6 \times 6$ supercell with a size of $37.80 \times 37.80 \times 180.35 \AA$ was used for $(R) /(S)$-3-quinuclidinol, including 1296 molecules and 28,512 atoms in total. The particle meshed ewald method was used for treating long-range electrostatic interaction $^{30}$ with a grid size of $1 \AA$, and the cutoff of Lennard-Jones ${ }^{31}$ and electrostatic interaction was set to $12 \AA$. During the simulation process, the time step was set to 2 fs.

A random Langevin dynamics thermostat ${ }^{32}$ and Nose-Hoover Langevin barostat were utilized for maintaining the constant temperature and pressure (NPT) ensemble, and the SHAKE algorithm ${ }^{33}$ was used for all covalent bonds involving hydrogen atoms. The steepest descent method was used to implement energy minimization for 60,000 steps, where the maximum force value of $10 \mathrm{~kJ} / \mathrm{nm} / \mathrm{mol}$ was taken as the convergence criterion. Then, 2 ns equilibrations were performed at the NPT ensemble. The equilibration simulations were run at a constant temperature of $300 \mathrm{~K}$ and a constant pressure of $1 \mathrm{~atm}$ with $2 \mathrm{~ns}$.

To obtain an equilibrium ferro-/paraelectric phase transition pathway, we performed 
replica-exchange molecular dynamics (REMD) simulations, ${ }^{34,} 35$ which can more effectively sample the conformational space. The "Multisander" package in the AMBER $20^{36}$ was used to deal with 36 replicas with a temperature range from 360 to $800 \mathrm{~K}$, each trajectory is obtained from a $10 \mathrm{~ns}$ production run. ${ }^{37}$ The strategy for selecting temperature is to first run a short REMD simulation in the experimental temperature and then calculate the temperature dependence of the average energy. To ensure that the system can pass the potential energy barriers, both the temperature values and the exchange frequency of temperatures of the replicas are controlled so that the acceptance rate of targets reaches $20 \%$ and remain constant during our simulations. ${ }^{35}$ The original structure was minimized via the first 500 cycles in the steepest descent method, and the SHAKE algorithm was utilized to constrain all the bonds with the hydrogen atoms, ${ }^{38}$ while adopting chiral constraint. Throughout the REMD simulations, initially, replicas were heated using Langevin thermostat for 200 ps, during which copy exchanges were attempted every 10,000 steps. ${ }^{39}$ The analysis was performed using the "ptraj" program in the Amber Tools software package. In addition, principal component analysis (PCA) was performed on the trajectory. First, the trajectories of 36 replicas were combined into a meta-trajectory for ensuring that the comparisons of all data were in the same subspace. Once the eigenvector was obtained, the meta-trajectory was projected onto the modes belonging two largest PCs. ${ }^{40}$ PCA was performed by $\mathrm{Cpptraj}^{41}$ in AMBER and standalone package Bio3D, ${ }^{42}$ and all visualization analysis is performed with VMD. ${ }^{43}$

\section{Results and discussion}

The comparison of the crystal structures of $(R)$-3-quinuclidinol and (S)-3-quinuclidinol can be found in Figure 1. The primitive cells of crystal structures of both $(R)$ - and $(S)$ - 3-quinuclidinol contain six molecules separately, with a long $c$-axis ( $z$-axis in the figure). Figure S1 and S2 present the ab initio energy band and density of states (DOS) of the $(R) /(S)$-3-quinuclidinol crystal under the HSE functional ${ }^{24}$, which shows a gap of $5.5 \mathrm{eV}$ and $5.7 \mathrm{eV}$, respectively, demonstrating the nature of the insulator. In its crystal, there are six molecules within a primitive cell, with a dipole moment of 2.45 Debye for both $(R)$ - and $(S)$ - enantiomers. Thus, the 
total dipole within the cell is $4.7 \mu \mathrm{C} \cdot \mathrm{cm}^{-2}$ in vacuum. The electrostatic polarization within the crystal provides about $2.0 \mu \mathrm{C} \cdot \mathrm{cm}^{-2}$ extra polarization, leaving a total spontaneous polarization of $6.7 \mu \mathrm{C} \cdot \mathrm{cm}^{-2}$.

a

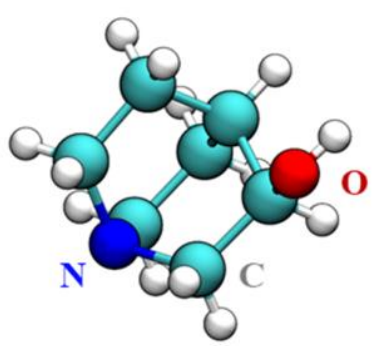

$\mathrm{b}$

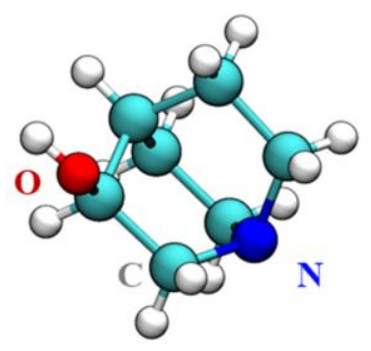

d
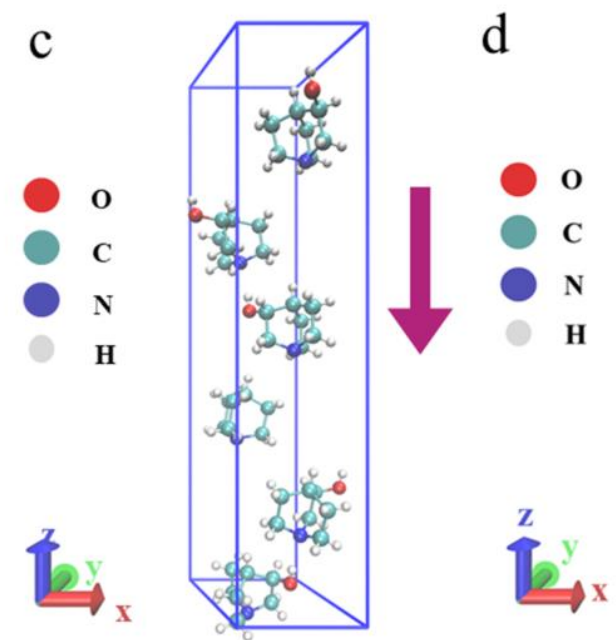

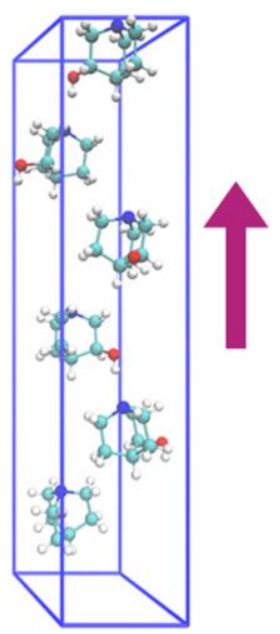

Figure 1: Single molecular and crystal structures of both enantiomers $(R)$-3-quinuclidinol and $(S)$-3-quinuclidinol.

a: Single molecule of $(R)$-3-quinuclidinol. b: Single molecule of $(S)$-3-quinuclidinol. c: Prime cell of (R)-3-quinuclidinol. d: Prime cell of (S)-3-quinuclidinol.

\section{Electric Hysteresis Loop}

To understand the origin of ferroelectricity of $(R) /(S)$-3-quinuclidinol, first we examined the changing of the crystal structure and polarization under the external electric field $\boldsymbol{E}$ at $300 \mathrm{~K}$. The direction of $\boldsymbol{E}$ is set along the $c$-axis, which is the $z$-direction in our model, and the magnitude of $\boldsymbol{E}$ is chosen as reported by experimental work ${ }^{15}$. During the MD simulation, $|\boldsymbol{E}|$ initially increased from 0 to 30 $\mathrm{kV} \mathrm{cm}{ }^{-1}$, then decreased to $-30 \mathrm{kV} \cdot \mathrm{cm}^{-1}$ inversely, and finally returned to $0 \mathrm{kV} \mathrm{cm}{ }^{-1}$ 
again.

The results are shown in Figure 2. It is clear that a fully aligned ferroelectric molecular arrangement forms after about $25 \mathrm{kV} \cdot \mathrm{cm}^{-1}$ and saturated to about $7.4 \pm$ $0.1 \mu \mathrm{C} \cdot \mathrm{cm}^{-2}$ for the $(R)$ - isomer and $7.8 \pm 0.1 \mu \mathrm{C} \cdot \mathrm{cm}^{-2}$ for the $(S)$ - isomer separately. and when the $\boldsymbol{E}$ reversed, the polarization switched accordingly to $-10 \mathrm{kV} \cdot \mathrm{cm}^{-1}$ and then reached a reversed saturate value after $|\boldsymbol{E}|$ increased to about $25 \mathrm{kV} \cdot \mathrm{cm}^{-1}$. Such results are highly compatible with experimental results, $P_{s}=6.69 \mu \mathrm{C} \cdot \mathrm{cm}^{-2}$ (for $(R)-$ ) and $P_{s}=6.72 \mu \mathrm{C} \cdot \mathrm{cm}^{-2}($ for $(S)-)$ at $303 \mathrm{~K}$. Our simulated values are also compatible to the values calculated by Xiong's group using first principles as $7 \mu \mathrm{C} \cdot \mathrm{cm}^{-2} .{ }^{15}$ From the simulated hysteresis loop, the $E_{\mathrm{c}}$ values for both $(R)$ - and $(S)$ - isomers are both 15 $\mathrm{kV} \cdot \mathrm{cm}^{-1}$, also highly consistent with experimental results of $15 \mathrm{kV} \cdot \mathrm{cm}^{-1}$. Due to the Landauer paradox ${ }^{44,45}$, our simulation would overestimate the $E_{\mathrm{c}}$.

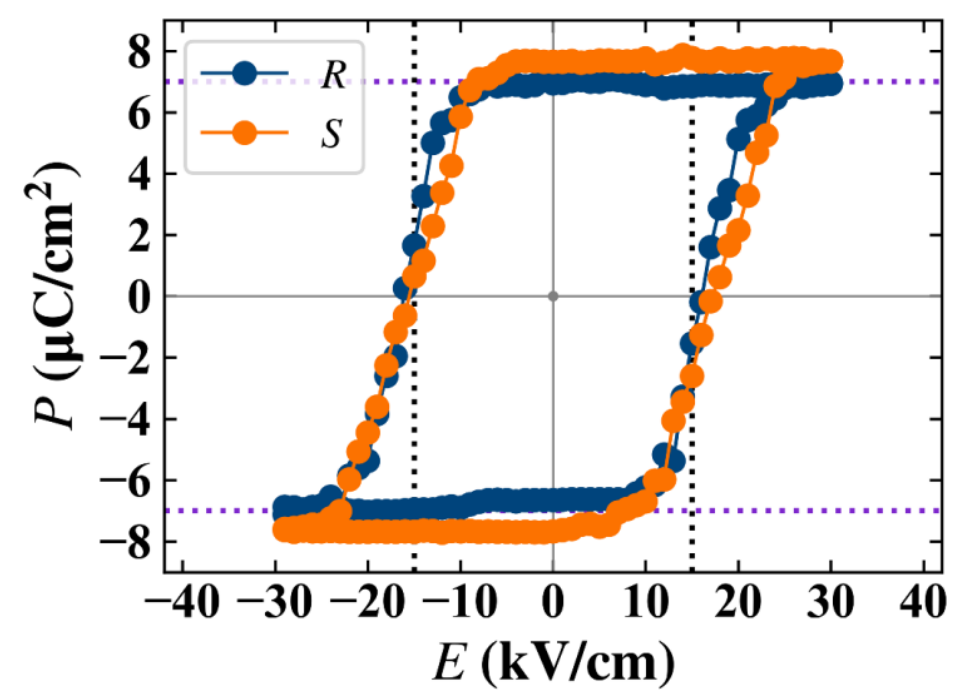

Figure 2: Ferroelectric hysteresis loop from MD simulation using PCC shows polarization switching of $(R) /(S)$-3-quinuclidinol under an external electrostatic field at $300 \mathrm{~K}$. From the hysteresis loop, the $P_{\mathrm{s}}$ values for $(R)$ and the $(S)$ - isomers are $7.4 \pm 0.1 \mu \mathrm{C} \cdot \mathrm{cm}^{-2}$ and $7.8 \pm 0.1 \mu \mathrm{C} \cdot \mathrm{cm}^{-2}$ respectively, and the $E_{\mathrm{c}}$ value for both of the isomers is $15 \mathrm{kV} \cdot \mathrm{cm}^{-1}$.

\section{Ferroelectric Switching Mechanism}


To reveal the switching mechanism, we also plot the $P_{\mathrm{s}}$ evolving with time. At the molecular level, the microscopic mechanism of the polarization switching of the $(R) /(S)$-3-quinuclidinol crystal material in the electric field is shown in Figure 3a, b. The structure consists of a $6 \times 6 \times 6$ supercell of the $(R) /(S)$-3-quinuclidinol crystal, which switches spontaneously from one orientation to the other at $300 \mathrm{~K}$ and keeps a constant volume under a coercive electric field of $15 \mathrm{kV} \cdot \mathrm{cm}^{-1}$ along the $c$-axis. The new domain starts nucleating at about $4.0 \mathrm{~ns}$, and the ferroelectric switching process is completed after 5.50 and $5.58 \mathrm{~ns}$, respectively for $(R)$ - and $(S)$ - isomers. The domain nuclei emerge randomly and grow quickly within about 0.2-0.3 ns, leaving most of the system switched, and then saturate gradually during another 0.3-0.5 ns. The total process occurs homogeneously in space and is lack of any pattern. but can be understood from Figure $3 \mathrm{a}$ and $\mathrm{b}$. Since in the snapshots, it can be seen that there are multiple micro domains occurring spontaneously after about $4 \mathrm{~ns}$, which violates one of the basic assumption of the Kolmogorov-Avrami-Ishibashi (KAI) model, that is, there is only a single domain existing during the whole switching process. ${ }^{46}$ 

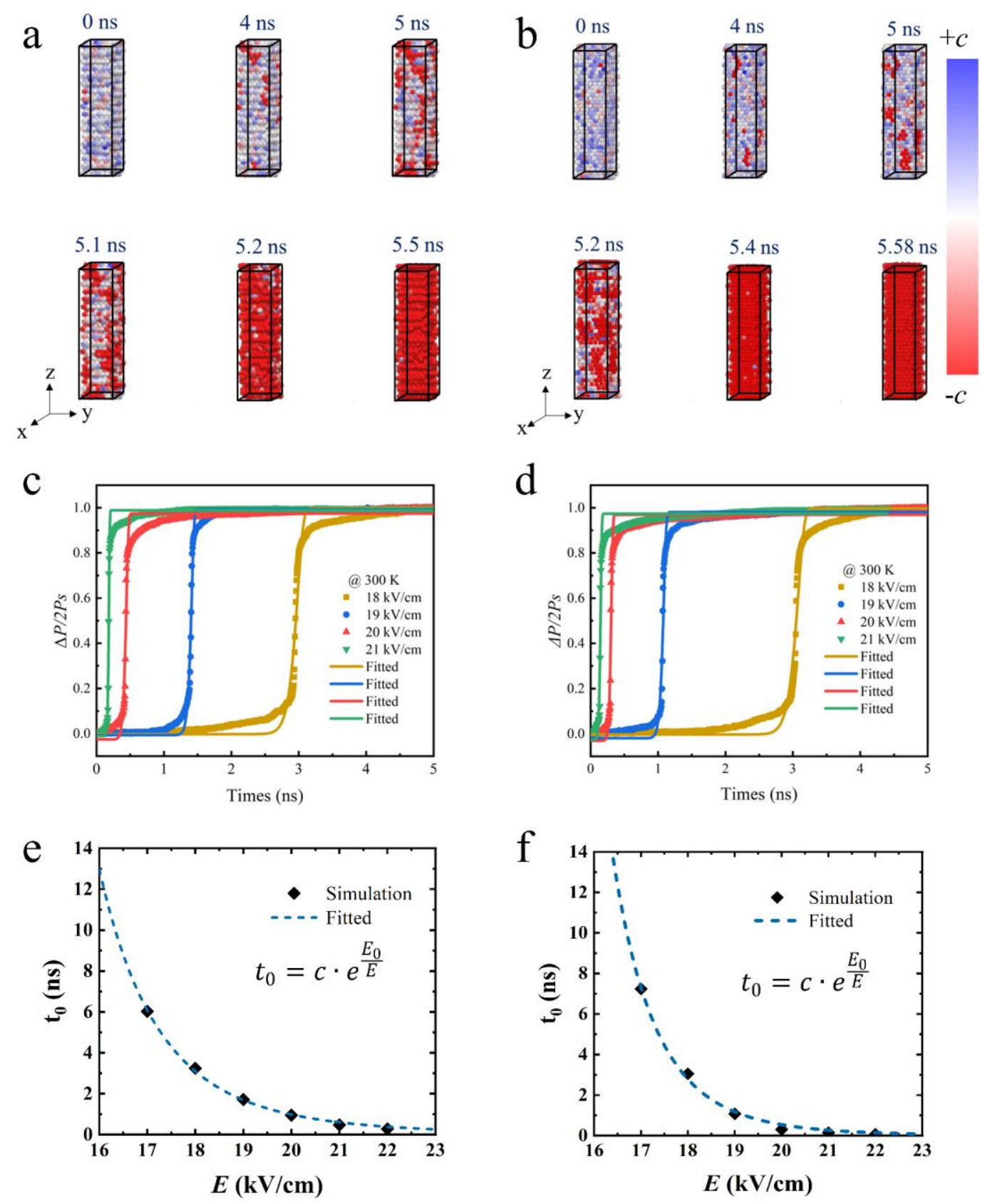

Figure 3: (a and b): Process of polarization switching of $(R) /(S)$-3-quinuclidinol in an external electric field of 17 $\mathrm{kV} \cdot \mathrm{cm}^{-1}$ under PCC MD simulation. The red and blue regions represent different directions of polarization, corresponding to domains with the $+c$ and $-c$ directions of their molecular dipoles. (a): $(R)$-3-quinuclidinol. (b): (S)-3-quinuclidinol. (c and d): The evolution of the growth of domain during the switching process under different $|\boldsymbol{E}|$. The solid lines correspond to fitted results using the KAI model. (e and f): Activation electric fields with the parameter $t_{0}$ fitted in the KAI model. The dashed lines represent fitted functions. 
For better understanding the switching mechanism, we fitted the evolution of spontaneous polarization with time to the (KAI) model, ${ }^{45,47}$ as did in our previous work $^{19}$ :

$$
\Delta P(t)=2 P_{s}\left(1-\mathrm{e}^{-\left(\frac{t}{t_{0}}\right)^{n}}\right)
$$

In the formula, the $t_{0}$ and $n$ are fitted parameters. The fitted lines comparing with the original data are shown in Figure 3(c-f). The converged parameters $t_{0}$ and $n$ are listed in Table S5. To our surprise, the switching mechanism differs much from the KAI model, especially in the time region of domain emerging and near saturating. However, in the asymptotic and switching region, the data are highly compatible with the KAI model. With the parameters from the KAI model, we also investigate the relationship between $t_{0}$ and the strength of the electric field $|\boldsymbol{E}|$, by fitting them to the Merz' law. ${ }^{48,49}$ These fittings indicate that the relationship between the characteristic conversion time of $(R) /(S)$-3-quinuclidinol and the external electric field is very consistent with that of traditional perovskite $\mathrm{BaTiO}_{3}{ }^{47,49}$ :

$$
t_{0}=c \mathrm{e}^{\frac{E_{0}}{E}}
$$

The fitting activation electric fields of $(R) /(S)$-3-quinuclidinol are $100.7 \mathrm{kV} \cdot \mathrm{cm}^{-1}$ and $157.7 \mathrm{kV} \cdot \mathrm{cm}^{-1}$, respectively. Here the data show high accordance to the model, showing a universality of such ferroelectric switching.

\section{3. phase Transition Mechanism}

Since the practical applications of ferroelectrics need maintaining the spontaneous polarization within a specific temperature range, we investigated its phase transition under changing of temperature. First, we performed MD simulations of 他和 $(R) /(S)$-3-quinuclidinol crystal in the temperature range of 100 to $800 \mathrm{~K}$, as shown in Figure 4. For (R)-3-quinuclidinol, all the trajectories converge within 2 ns. And when the temperature gradually gets close to $370 \pm 4 \mathrm{~K}$, the average value of polarization decreases with the fluctuation increasing. When the temperature reaches $375 \pm 3 \mathrm{~K}$, the polarization drops suddenly to $1.0 \mu \mathrm{C} \cdot \mathrm{cm}^{-2}$ at the very beginning indicating that 
the ferroelectric phase transforms into paraelectric one. Within fluctuation of MD simulation, it shows a first-order phase transition. Such finding confirms the results from second harmonic generation experiments. ${ }^{15}$ However, this protocol fails to give reasonable $T_{c}$ for $(S)$-3-quinuclidinol, which shows a quite high $T_{c}$ as $700 \mathrm{~K}$, far from the reported value of $400 \mathrm{~K}$. It would stem from the fact that the free energy barrier for the phase transition of $(S)$-3-quinuclidinol is too high to allow the system to overcome it at $400 \mathrm{~K}$.
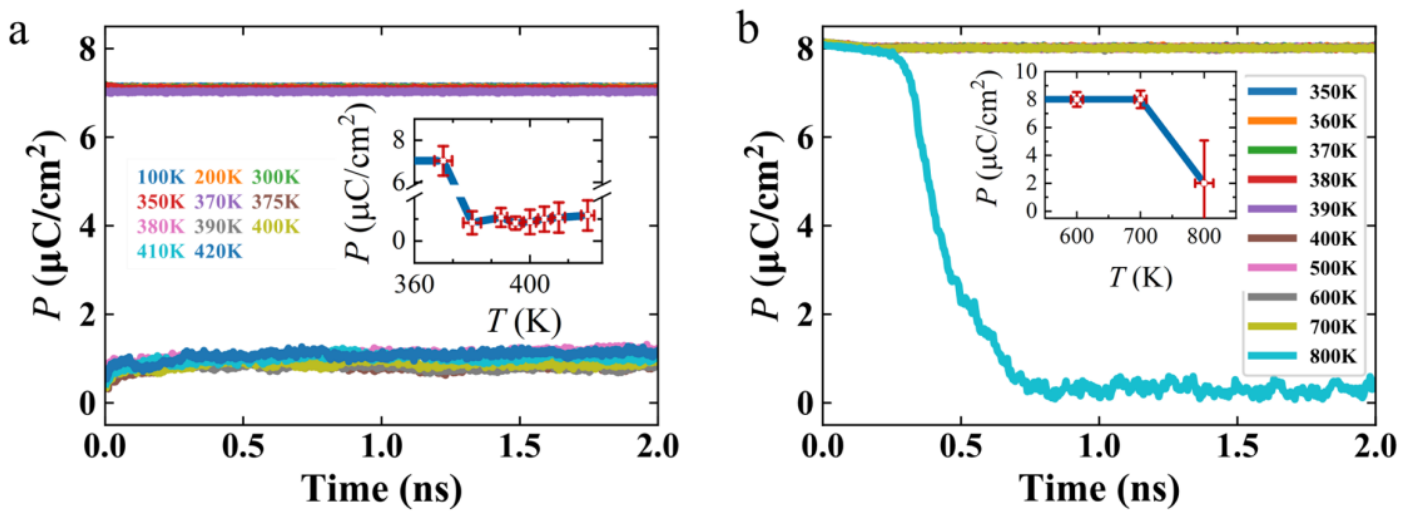

Figure 4: Temperature dependence curve of (a) (R)-3-quinuclidinol and (b) (S)-3-quinuclidinol. The subplot shows the polarization evolving with temperature.

To verify our consideration and obtain reliable $T_{\mathrm{c}}$, we performed enhanced sampling replica-exchange REMD simulations to find the reasonable $T_{\mathrm{c}}$ by effective sampling in phase space ${ }^{55}$. 

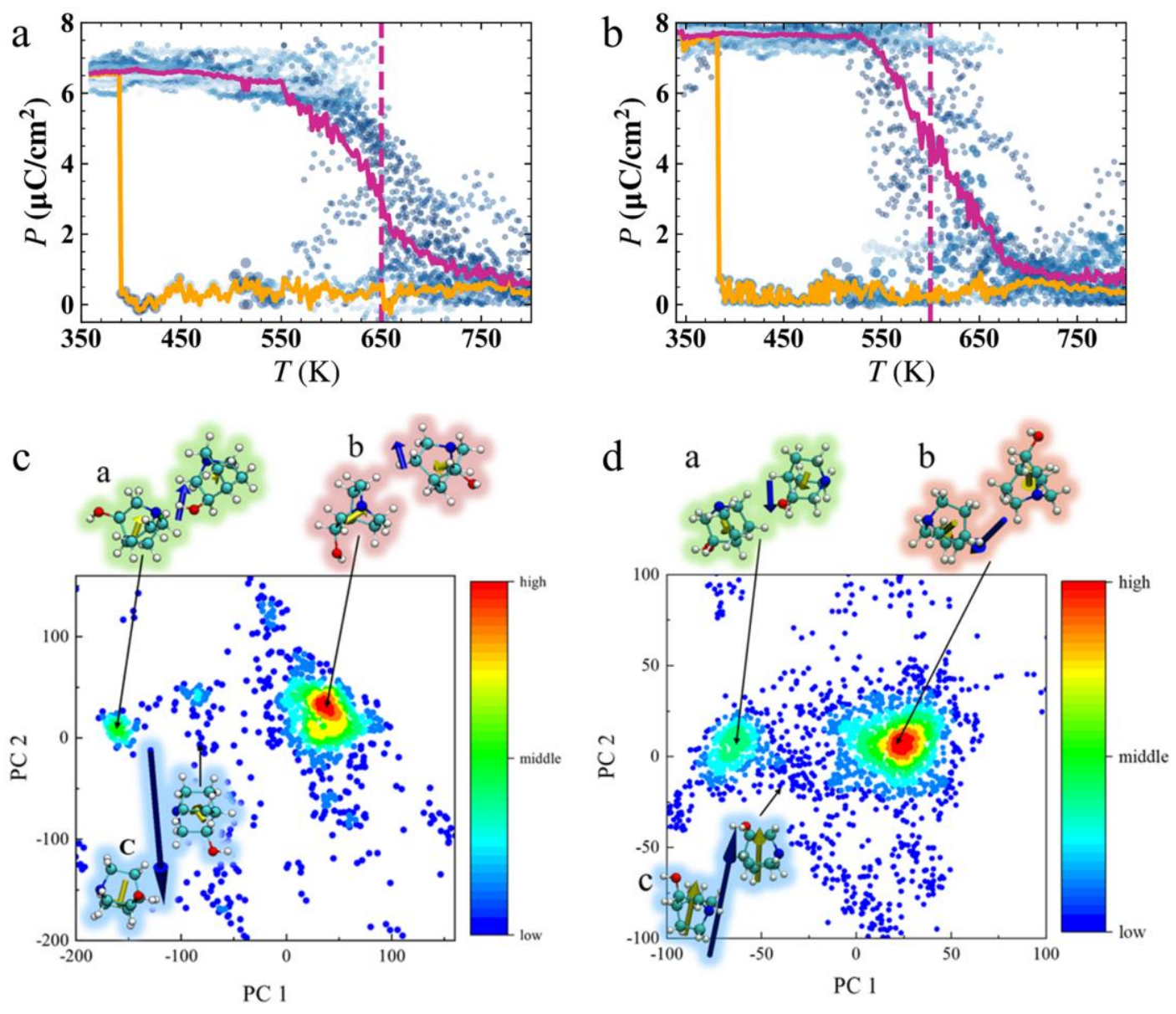

Figure 5: (a and b) : Phase transition behaviors of $(R) /(S)$-3-quinuclidinol. The yellow line shows the first phase transitions at $400 \mathrm{~K}$ of $(R)$-3-quinuclidinol and $398 \mathrm{~K}$ of $(S)$-3-quinuclidinol, which are highly consistent with the experimental values, and the purple line shows the other phase transition at a high temperature of about $600 \mathrm{~K}$. (c and d): PCA for $(R)$ - and $(S)$ - isomers. The color bar shows the density of the points representing structures, and arrowed points (a) and (b) represent the characteristic structures for two paraelectric phases, and (c) represents the characteristic structure for the ferroelectric phase.

Figure 5a, b exhibits the spontaneous polarization of the $(R) /(S)$-3-quinuclidinol crystal with respect to temperature from REMD. Surprisingly, they both show two different critical points, $380 \mathrm{~K}(R)-/ 385 \mathrm{~K}(S)$ - and $650 \mathrm{~K}(R)-/ 600 \mathrm{~K}(S)$-. The first one of each isomer is definitely the $T_{\mathrm{c}}$ found from experimental work ${ }^{14}$. The second one is a novel finding since it has not been reported by experiments yet. Howeversince the decomposition temperatures for the crystals of both $(R)$ - and $(S)$ - 
isomers are about $495 \mathrm{~K}$, it would be a challenge for experimentalists to validate the second critical point. For validating our finding, we also performed cluster analysis forPCs, as shown in Figure 5c, d. Figures S7 and S8 display the proportion of the PCs in the variance of the original distribution. ${ }^{39}$ The scattered points represent the structures from all of the REMD calculations described by PCs, and the color is produced by kernel density estimation. From the plots, it can be recognized that there are three major clusters for each isomer, denoted by a, b, and c. We display the characteristic structure of each cluster by picking the center of the highest density in each cluster in Figure 5c, d. The cluster $\mathrm{c}$ for each isomer is in the beginning ferroelectric phase, and both a and c are in the paraelectric phase. Therefore, it is clear that there are two different paraelectric phases corresponding to both $T_{\mathrm{c}}$. 

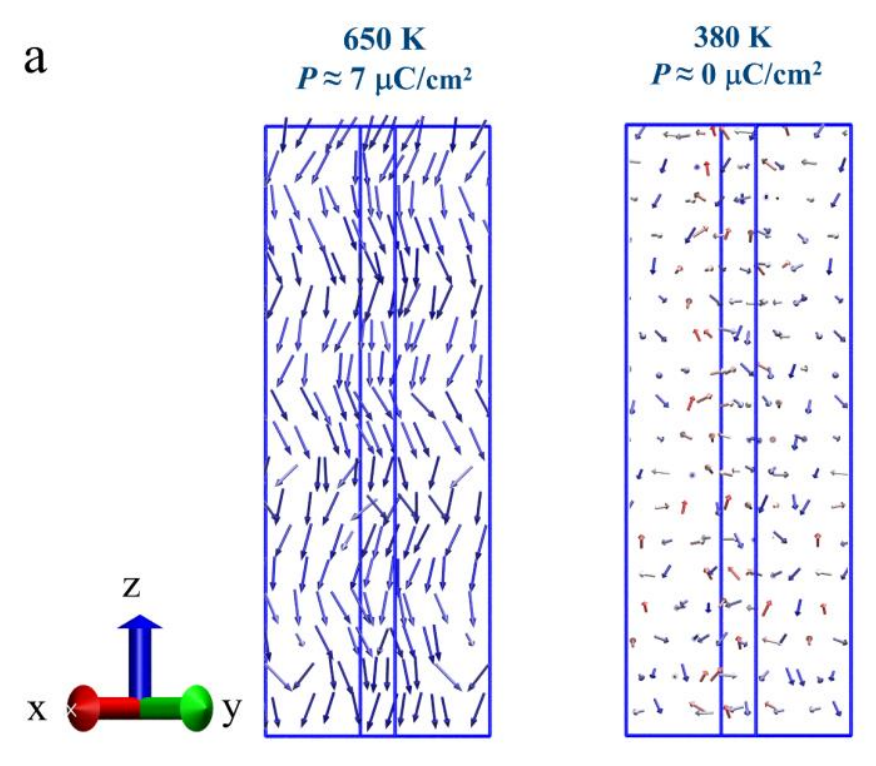

$650 \mathrm{~K}$
$P \approx 0 \mu \mathrm{C} / \mathrm{cm}^{2}$
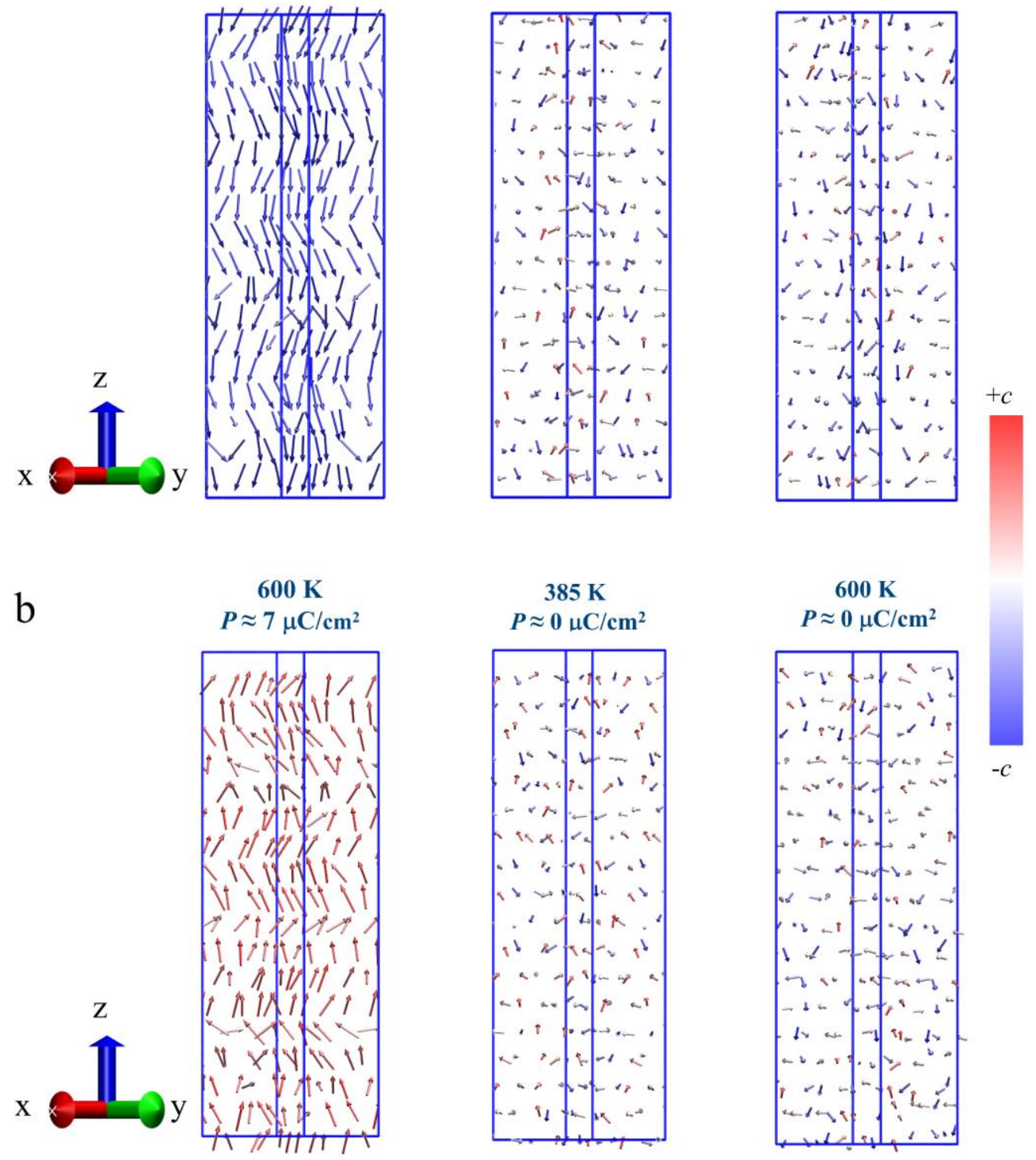

Figure 6: Dipole moments of each molecule in the three conformations of the $(R) /(S)$-3-quinuclidinol crystal are

shown by arrows. (a): (R)-3-quinuclidinol. (b): (S)-3-quinuclidinol. From left to right: the ferroelectric phase, the paraelectric phase (a), and the paraelectric phase (b). Blue represents the negative direction along the $c$-axis, and red represents the positive direction along the $c$-axis.

For differentiating each phase, we also depicted dipole moments of six molecules within prime cells for all the three characteristic conformations. ${ }^{5}$ As shown in Figure 6, the structures and corresponding dipole moments are shown alongside the clusters. The blue arrow represents the dipole moment of a single cell, and the yellow one 
represents the dipole moment of each molecule. All the three characteristic conformations are analyzed by Multiwfn. ${ }^{50}$ The structures and corresponding dipole moments are shown alongside the clusters. Details are shown in Figures S5 and S6 and Tables S3 and S4. From these plots, we can understand that for both the paraelectric phases, the dipole moment within each prime cell is non-zero but randomly arranged, resulting in a total paraelectric phase. However, the difference between both of the paraelectric phases is hard to distinguish by eyes. By using the results of REMD, we also calculated the potential of mean force (PMF) of the phase transition using the multiple Bennett's acceptance ratio (MBAR) method. ${ }^{51,52}$ The free energy barrier for the $(S)$ - enantiomer is $6.7 \mathrm{kcal} / \mathrm{mol}$, slightly higher than that of the $(R)-, 6.0 \mathrm{kcal} / \mathrm{mol}$. The profile is shown in Figure 7. Since the difference between both barriers is small, we contribute the difficulty of phase transition of the $(S)$ enantiomer to the broad width of PMF. However, a small increase in its height hinders the system much to overcome it.

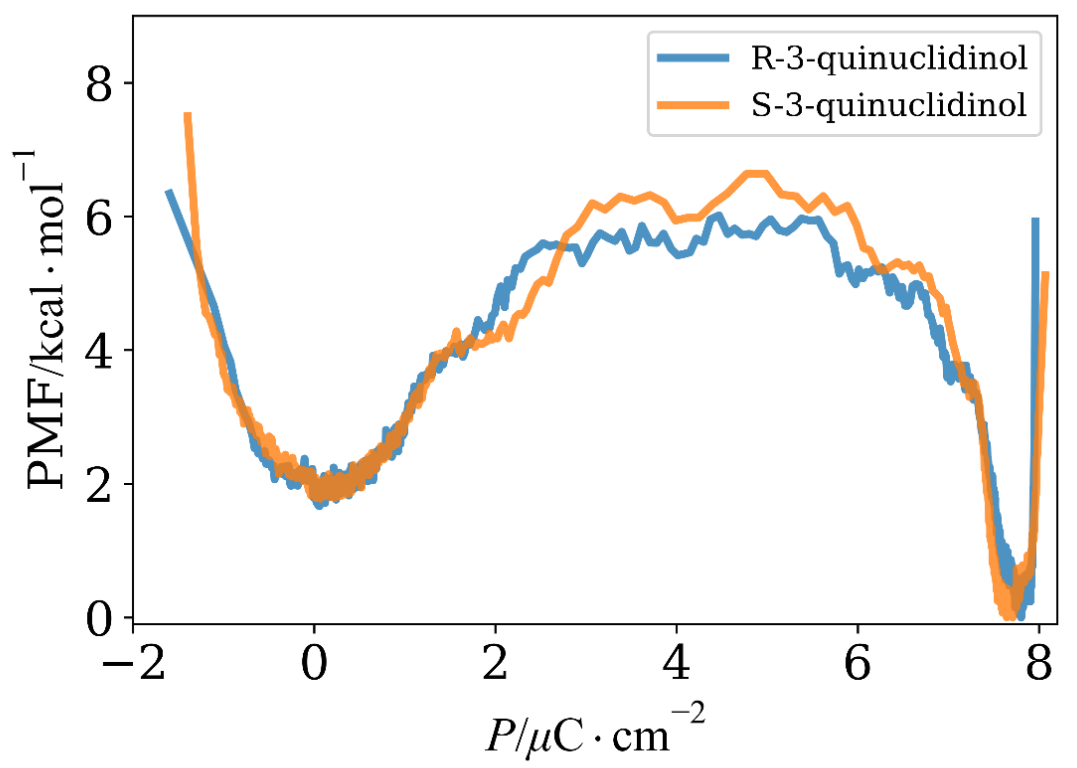

Figure 7: PMFobtained from MBAR calculations using REMD results, at the first $T_{\mathrm{c}}, 380 \mathrm{~K}$.

\section{Conclusion}

We investigated the origin of spontaneous polarization, the mechanism of both ferroelectric switching and phase transition for $(R) /(S)$-3-quinuclidinol, based on MD simulations with PCC. Our results validate the $T_{\mathrm{c}}$ and $E_{\mathrm{c}}$ for the crystals of both 
isomers, as high as $380 \mathrm{~K}(R)-/ 385 \mathrm{~K}(S)$-, which is much higher than other chiral ferroelectrics and comparable to the classic inorganic ferroelectric barium titanate (393 K). In the study of $T_{\mathrm{c}}$, we also find a novel critical point as high as about $600 \mathrm{~K}$, higher than the decomposition temperature of $(R) /(S)$-3-quinuclidinol. We also find that the switching process is not according to the KAI model; hoping future work can provide a new model to explain it. In addition, the saturated polarization strength (up to $8 \mu \mathrm{C} \cdot \mathrm{cm}^{-2}$ ) is equivalent to the organic polymer ferroelectric $\mathrm{PVDF}^{55}$, and the low coercive field $\left(15 \mathrm{kV} \cdot \mathrm{cm}^{-1}\right)$ can ensure that the ferroelectric polarization is easy to switch. This result reveals that $(R) /(S)$-3-quinuclidinol has good ferroelectricity, which is also necessary for ferroelectric devices.

\section{Competing interests}

The authors declare no competing interests.

\section{Corresponding author}

Correspondence to Yongle Li.

\section{Acknowledgments}

This study was funded by the National Natural Science Foundation of China (No. 21503130 and 11674212). We appreciate the Ziqiang 4000 of Shanghai University for high-performance computing services. We thank Ye Mei (East China Normal University) and Ya Gao (Shanghai University of Engineering Science) for some helpful discussions.

Supporting Information: The detailed crystal structures and the density of states of $(R) /(S)$-3-quinuclidinol crystal and the simulation results of dynamic simulation. 


\section{References}

1. Asadi, K.; Li, M.; Blom, P. W. M.; Kemerink, M.; de Leeuw, D. M., Organic Ferroelectric Opto-Electronic Memories. Mater. Today 2011, 14, 592-599.

2. Scott, J. F., Applications of Modern Ferroelectrics. Science 2007, 315, 954-959.

3. Martin, L. W.; Rappe, A. M., Thin-Film Ferroelectric Materials and Their Applications. Nat. Rev. Mater. 2016, 2, 16087.

4. Kaminow, I., Principles and Applications of Ferroelectrics and Related Materials. Phys. Today 1978, 31, 56-58.

5. Valasek, J., Piezo-Electric and Allied Phenomena in Rochelle Salt. Phys. Rev. 1921, 17, 475-481.

6. Chen, H. D.; Udayakumar, K. R.; Cross, L. E.; Bernstein, J. J.; Niles, L. C., Dielectric, Ferroelectric, and Piezoelectric Properties of Lead Zirconate Titanate Thick Films on Silicon Substrates. J. Appl. Phys. 1995, 77, 3349-3353.

7. Zhao, Z.; Buscaglia, V.; Viviani, M.; Buscaglia, M. T.; Mitoseriu, L.; Testino, A.; Nygren, M.; Johnsson, M.; Nanni, P., Grain-Size Effects on the Ferroelectric Behavior of Dense Nanocrystalline Batio 3 Ceramics. Phys. Rev. B 2004, 70, 024107.

8. Horiuchi, S.; Ishibashi, S., Hydrogen-Bonded Small-Molecular Crystals Yielding Strong Ferroelectric and Antiferroelectric Polarizations. J. Phys. Soc. Japan 2020, 89, 051009.

9. Meyer, R. B.; Liebert, L.; Strzelecki, L.; Keller, P., Ferroelectric Liquid Crystals. J. Physique Lett. 1975, 36, 69-71.

10. A Century of Ferroelectricity. Nat. Mater. 2020, 19, 129-129.

11. Horiuchi, S.; Kagawa, F.; Hatahara, K.; Kobayashi, K.; Kumai, R.; Murakami, Y.; Tokura, Y. J. N.

C., Above-Room-Temperature Ferroelectricity and Antiferroelectricity in Benzimidazoles. Nat. Commun. 2012, 3, 187-190.

12. Ye, H.-Y.; Tang, Y.-Y.; Li, P.-F.; Liao, W.-Q.; Gao, J.-X.; Hua, X.-N.; Cai, H.; Shi, P.-P.; You, Y.-M.; Xiong, R.-G., Metal-Free Three-Dimensional Perovskite Ferroelectrics. Science 2018, 361, 151-155.

13. Liao, W.-Q.; Zhao, D.; Tang, Y.-Y.; Zhang, Y.; Li, P.-F.; Shi, P.-P.; Chen, X.-G.; You, Y.-M.; Xiong, R.-G., A Molecular Perovskite Solid Solution with Piezoelectricity Stronger Than Lead Zirconate Titanate. Science 2019, 363, 1206-1210.

14. Wang, Z.-X.; Zhang, H.; Wang, F.; Cheng, H.; He, W.-H.; Liu, Y.-H.; Huang, X.-Q.; Li, P.-F., Superior Transverse Piezoelectricity in a Halide Perovskite Molecular Ferroelectric Thin Film. J. Am. Chem. Soc. 2020, 142, 12857-12864.

15. Li, P. F.; Liao, W. Q.; Tang, Y. Y.; Qiao, W.; Zhao, D.; Ai, Y.; Yao, Y. F.; Xiong, R. G., Organic Enantiomeric High-T C Ferroelectrics. Proc. Natl. Acad. Sci. U.S.A. 2019, 116, 5878-5885.

16. Sun, Z.; Chen, T.; Luo, J.; Hong, M., Bis(Imidazolium) L-Tartrate: A Hydrogen-Bonded Displacive-Type Molecular Ferroelectric Material. Angew. Chem. Int. Ed. Engl. 2012, 51, 3871-3876.

17. Blázquez-Castro, A.; García-Cabañes, A.; Carrascosa, M., Biological Applications of Ferroelectric Materials. Appl. Phys. Rev. 2018, 5.

18. Qi, L.; Ruan, S.; Zeng, Y.-J., Review on Recent Developments in 2d Ferroelectrics: Theories and Applications. Adv. Mater. 2021, 33, 2005098.

19. Hu, P.; Hu, S.; Huang, Y.; Reimers, J. R.; Rappe, A. M.; Li, Y.; Stroppa, A.; Ren, W., Bioferroelectric Properties of Glycine Crystals. J. Phys. Chem. Lett. 2019, 10, 1319-1324.

20. Allen, F. H., et al., The Cambridge Crystallographic Data Centre: Computer-Based Search, Retrieval, Analysis and Display of Information. Acta Crystallogr., Sect. B 1979, 35, 2331-2339. 
21. Hafner, J., Ab-Initio Simulations of Materials Using Vasp: Density-Functional Theory and Beyond. J. Comput. Chem. 2008, 29, 2044-78.

22. Kresse, G.; Joubert, D., From Ultrasoft Pseudopotentials to the Projector Augmented-Wave Method. Phys. Rev. B 1999, 59, 1758-1775.

23. Perdew, J. P.; Burke, K.; Ernzerhof, M., Generalized Gradient Approximation Made Simple. Phys. Rev. Lett. 1996, 77, 3865-3868.

24. Heyd, J.; Scuseria, G. E., Efficient Hybrid Density Functional Calculations in Solids: Assessment of the Heyd-Scuseria-Ernzerhof Screened Coulomb Hybrid Functional. J. Chem. Phys. 2004, 121, 1187-1192.

25. Momma, K.; Izumi, F., Vesta 3 for Three-Dimensional Visualization of Crystal, Volumetric and Morphology Data. J. Appl. Crystallogr. 2011, 44, 1272-1276.

26. Huang, Y.; Hu, P.; Song, J.; Li, Y.; Stroppa, A., Molecular Dynamics Simulations of Ferroelectricity in Di-Isopropyl-Ammonium Halide Molecular Crystals. Chem. Phys. Lett. 2019, 730, 367-371.

27. Humphrey, W.; Dalke, A.; Schulten, K., Vmd: Visual Molecular Dynamics. J. Mol. Graph. 1996, 14, 33-38.

28. Nelson, M. T.; Humphrey, W.; Gursoy, A.; Dalke, A.; Kalé, L. V.; Skeel, R. D.; Schulten, K., Namd: A Parallel, Object-Oriented Molecular Dynamics Program. IJHPCA 1996, 10, 251-268.

29. Phillips, J. C.; Braun, R.; Wang, W.; Gumbart, J.; Tajkhorshid, E.; Villa, E.; Chipot, C.; Skeel, R. D.; Kalé, L.; Schulten, K., Scalable Molecular Dynamics with Namd. J. Comput. Chem. 2005, 26, 1781-1802.

30. Nam, K.; Gao, J.; York, D. M., An Efficient Linear-Scaling Ewald Method for Long-Range Electrostatic Interactions in Combined Qm/Mm Calculations. J. Chem. Theory Comput. 2005, 1, 2-13.

31. Hansen, J.-P.; Verlet, L., Phase Transitions of the Lennard-Jones System. Phys. Rev. 1969, 184, 151-161.

32. Pastor, R.; Brooks, B.; AttilaSzabo, An Analysis of the Accuracy of Langevin and Molecular Dynamics Algorithms. Mol. Phys. 2006, 65, 1409-1419.

33. Ryckaert, J. P.; Ciccotti, G.; Berendsen, H. J. C., Numerical Integration of the Cartesian Equations of Motion of a System with Constraints: Molecular Dynamics of N-Alkanes. J. Comput. Phys. 1977, 23, 327-341.

34. Zhang, W.; Wu, C.; Duan, Y., Convergence of Replica Exchange Molecular Dynamics. J. Chem. Phys. 2005, 123, 154105 .

35. Rathore, N.; Chopra, M.; de Pablo, J. J., Optimal Allocation of Replicas in Parallel Tempering Simulations. J. Chem. Phys. 2004, 122, 024111.

36. Salomon-Ferrer, R.; Case, D. A.; Walker, R. C., An Overview of the Amber Biomolecular Simulation Package. Wiley Interdiscip. Rev. Comput. Mol. Sci. 2013, 3, 198-210.

37. Abraham, M. J.; Gready, J. E., Ensuring Mixing Efficiency of Replica-Exchange Molecular Dynamics Simulations. J. Chem. Theory Comput. 2008, 4, 1119-1128.

38. Sugita, Y.; Okamoto, Y., Replica-Exchange Molecular Dynamics Method for Protein Folding. Chem. Phys. Lett. 1999, 314, 141-151.

39. Song, J.; Li, Y.; Ji, C.; Zhang, J. Z., Functional Loop Dynamics of the Streptavidin-Biotin Complex. Sci. Rep. 2015, 5, 7906.

40. Roe, D. R.; Cheatham, T. E., Ptraj and Cpptraj: Software for Processing and Analysis of Molecular Dynamics Trajectory Data. J. Chem. Theory Comput. 2013, 9, 3084-3095. 
41. Grant, B. J.; Rodrigues, A. P.; ElSawy, K. M.; McCammon, J. A.; Caves, L. S., Bio3d: An R Package for the Comparative Analysis of Protein Structures. Bioinformatics 2006, 22, 2695-6.

42. Hilt, S.; Shabbir, S.; Anders, J.; Lutz, E., Landauer's Principle in the Quantum Regime. Phys. Rev. E 2011, 83, 030102.

43. Jiang, A. Q.; Lee, H. J.; Hwang, C. S.; Tang, T. A., Resolving the Landauer Paradox in Ferroelectric Switching by High-Field Charge Injection. Phys. Rev. B 2009, 80, 024119.

44. Tagantsev, A. K.; Stolichnov, I.; Setter, N.; Cross, J. S.; Tsukada, M., Non-Kolmogorov-Avrami Switching Kinetics in Ferroelectric Thin Films. Phys. Rev. B 2002, 66.

45. Jo, J. Y.; Han, H. S.; Yoon, J. G.; Song, T. K.; Kim, S. H.; Noh, T. W., Domain Switching Kinetics in Disordered Ferroelectric Thin Films. Phys. Rev. Lett. 2007, 99, 267602.

46. Boddu, V.; Endres, F.; Steinmann, P., Molecular Dynamics Study of Ferroelectric Domain Nucleation and Domain Switching Dynamics. Sci. Rep. 2017, 7, 806.

47. Miller, R. C.; Weinreich, G., Mechanism for the Sidewise Motion of $180^{\circ}$ Domain Walls in Barium Titanate. Phys. Rev. 1960, 117, 1460-1466.

48. Zhao, D.; Lenz, T.; Gelinck, G. H.; Groen, P.; Damjanovic, D.; de Leeuw, D. M.; Katsouras, I., Depolarization of Multidomain Ferroelectric Materials. Nat. Commun. 2019, 10, 2547.

49. Shin, Y. H.; Grinberg, I.; Chen, I. W.; Rappe, A. M., Nucleation and Growth Mechanism of Ferroelectric Domain-Wall Motion. Nature 2007, 449, 881-4.

50. Lu, T.; Chen, F., Multiwfn: A Multifunctional Wavefunction Analyzer. J. Comput. Chem. 2012, 33, 580-592.

51. Liu, W.; Li, P.; Mei, Y., Discovery of Sbf1 as an Allosteric Inhibitor Targeting the Pif-Pocket of 3-Phosphoinositide-Dependent Protein Kinase-1. J. Mol. Model. 2019, 25, 187.

52. Shirts, M. R.; Chodera, J. D., Statistically Optimal Analysis of Samples from Multiple Equilibrium States. J. Chem. Phys. 2008, 129, 124105.

53. Kliem, H., Monte Carlo Simulations of Ferroelectric Properties for Pvdf and Batio. Ferroelectrics 2008, 370, 207-218. 
Supporting Information for

\section{Mechanisms of Molecular Ferroelectrics Made Simple}

Xiaoqing Zhu, ${ }^{a b}$ Wenbin Fan, ${ }^{a b}$ Wei Ren, ${ }^{a b}$ and Yongle Li*ab

a Department of Physics, Shanghai University, Shanghai 200444, China.

${ }^{\mathrm{b}}$ Department of Physics, International Center of Quantum and Molecular Structures, Shanghai University, Shanghai 200444, China.

*Email: yongleli@shu.edu.cn; 

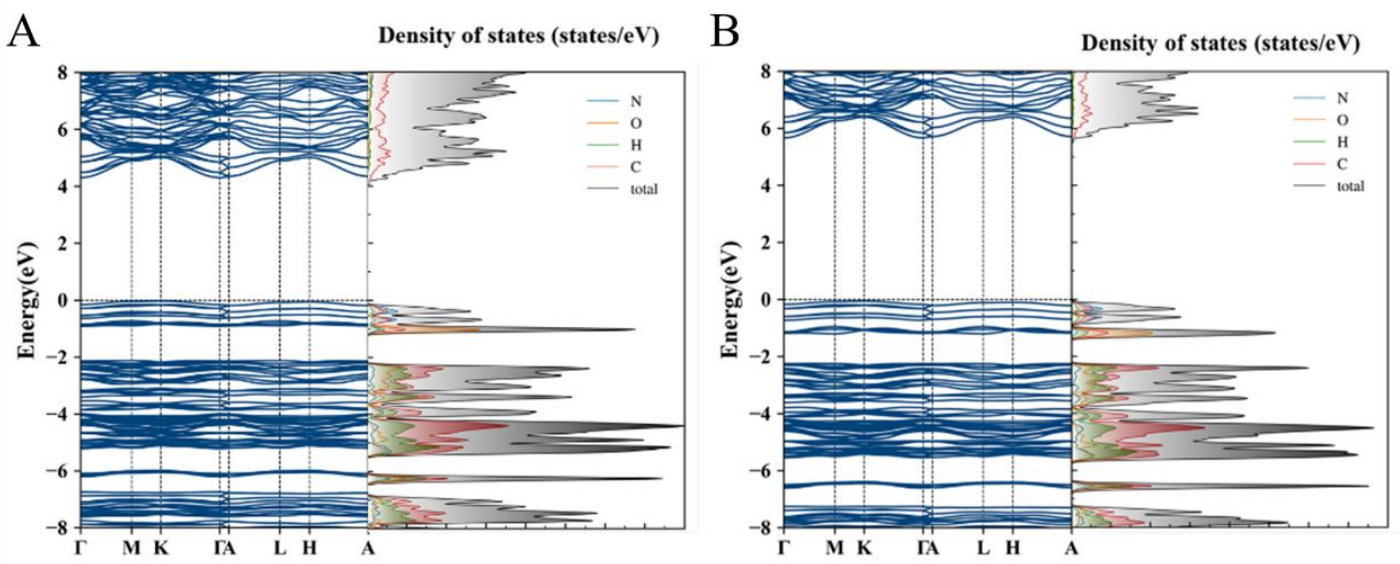

Figure S1: The density of states (DOS) of $R$-3-quinuclidinol calculated through (A) PBE exchange-correlation functionals and (B) Heyd-Scuseria-Ernzerhof functionals. The contributions from nitrogen, oxygen, hydrogen, and carbon atoms are highlighted by blue, orange, green, and purple lines, respectively.
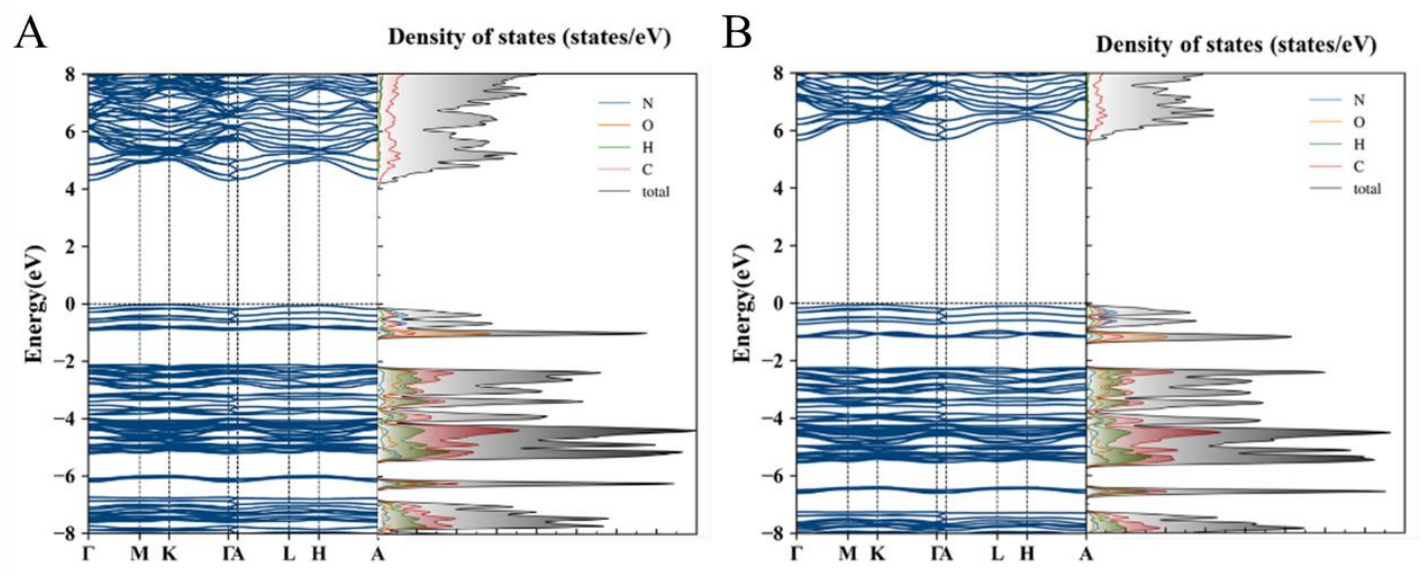

Figure S2: The density of states (DOS) of $S$-3-quinuclidinol as calculated through (A) PBE exchange-correlation functionals and (B) Heyd-Scuseria-Ernzerhof functionals. The contributions from nitrogen, oxygen, hydrogen, and carbon atoms are highlighted by blue, orange, green, and purple lines, respectively. 


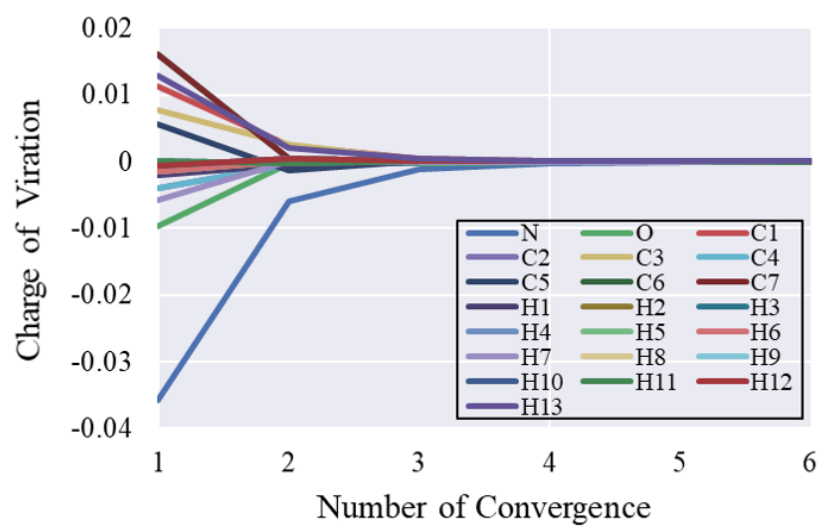

Figure S3: The PCC charge convergence of $(R)$-3-quinuclidinol crystal.

Table S1. The charge convergence of $(R)$-3-quinuclidinol crystal

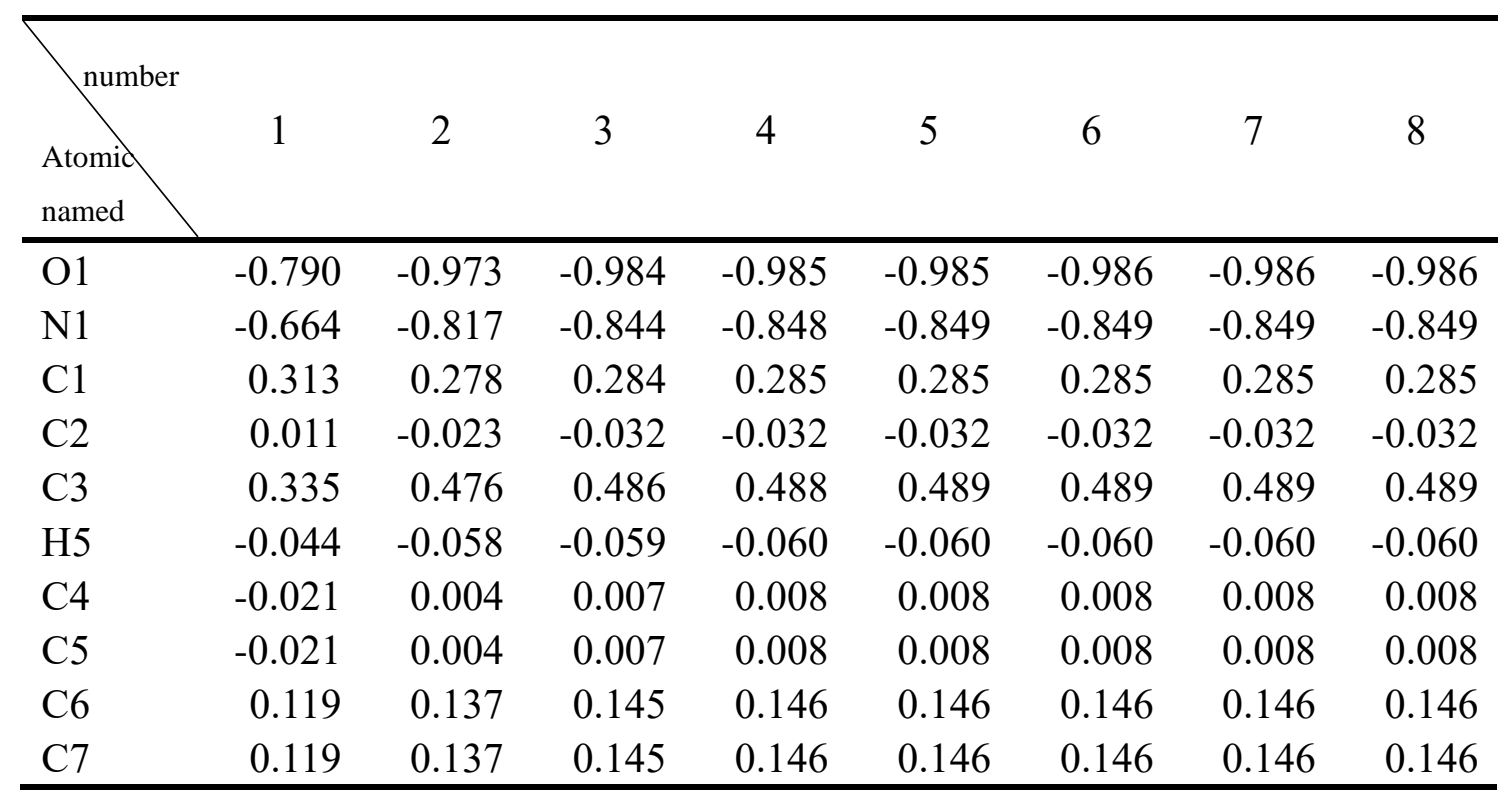




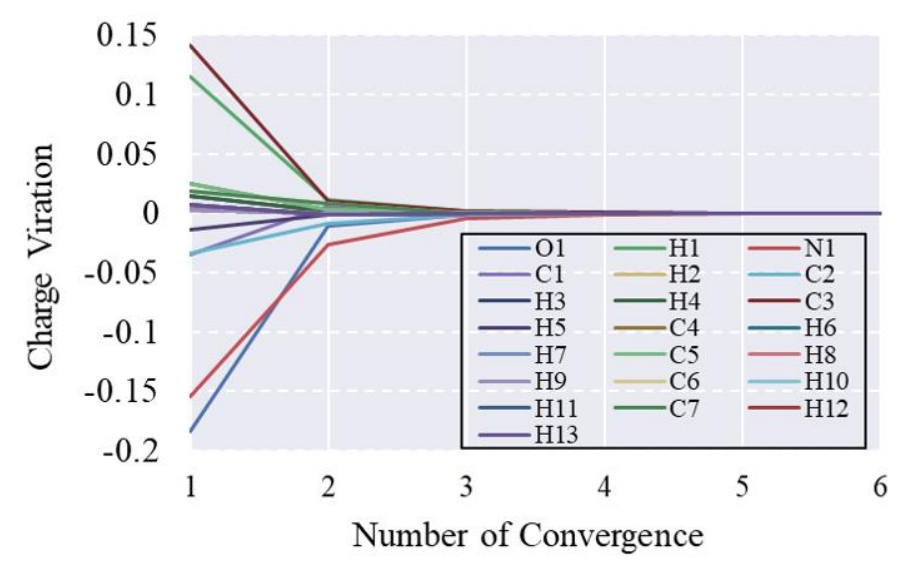

Figure S4: The PCC charge convergence of (S)-3-quinuclidinol.

Table S2. The charge convergence of $(S)$-3-quinuclidinol crystal

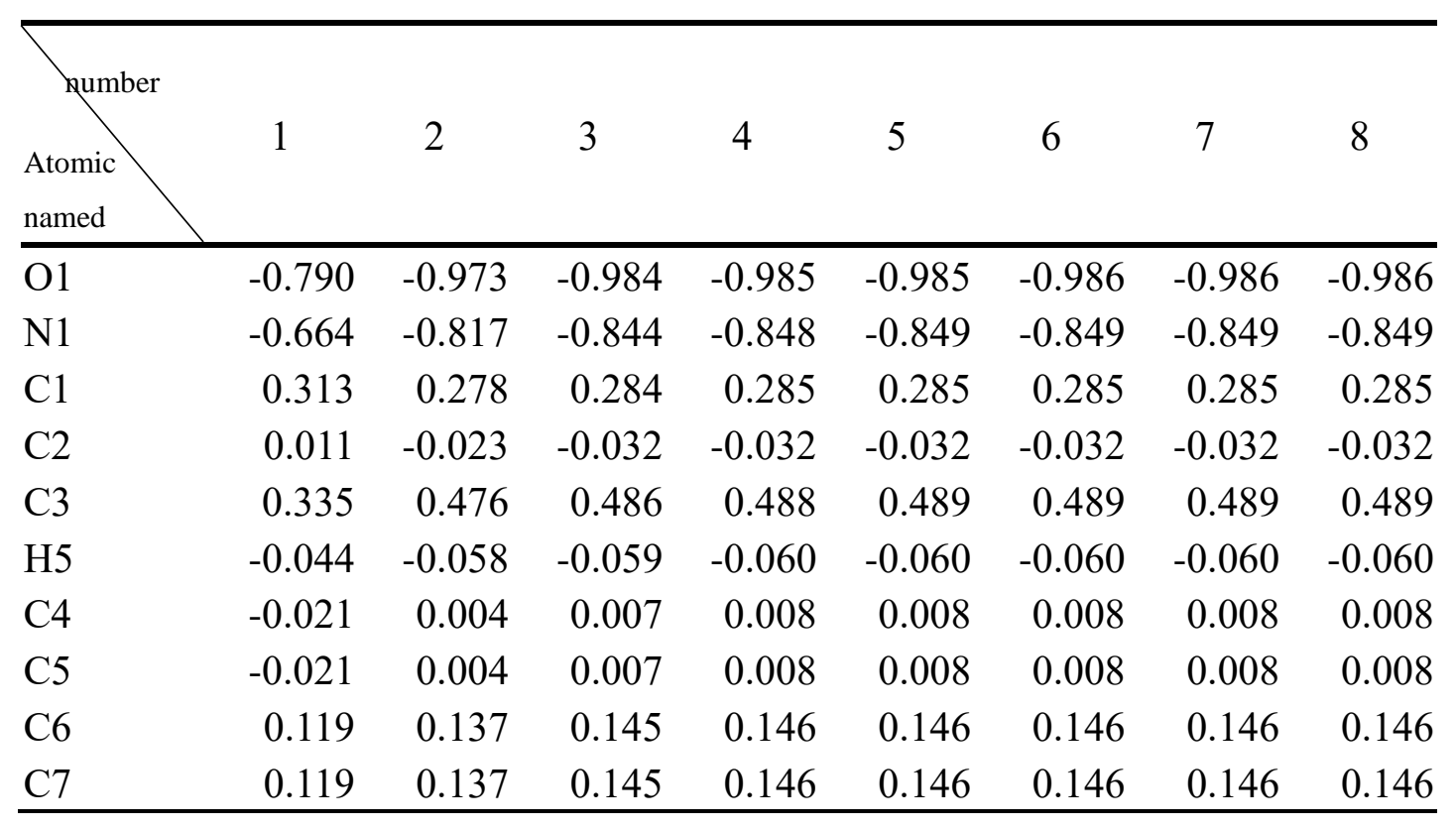


Table S3. Acceptance ratios of replica corresponding to pairs of neighboring temperatures for $(R)$-3-quinuclidinol crystal.

\begin{tabular}{lc|cc}
\hline Pair of temperatures & Acceptance ratio & Pair of temperatures & Acceptance ratio \\
\hline $350.00 \mathrm{~K} \leftrightarrow 358.18 \mathrm{~K}$ & 0.263 & $544.59 \mathrm{~K} \leftrightarrow 556.28 \mathrm{~K}$ & 0.263 \\
$358.18 \mathrm{~K} \leftrightarrow 366.50 \mathrm{~K}$ & 0.239 & $556.28 \mathrm{~K} \leftrightarrow 568.18 \mathrm{~K}$ & 0.248 \\
$366.50 \mathrm{~K} \leftrightarrow 374.97 \mathrm{~K}$ & 0.251 & $568.18 \mathrm{~K} \leftrightarrow 580.29 \mathrm{~K}$ & 0.261 \\
$374.97 \mathrm{~K} \leftrightarrow 383.59 \mathrm{~K}$ & 0.264 & $580.29 \mathrm{~K} \leftrightarrow 592.62 \mathrm{~K}$ & 0.257 \\
$383.59 \mathrm{~K} \leftrightarrow 392.37 \mathrm{~K}$ & 0.274 & $592.62 \mathrm{~K} \leftrightarrow 605.18 \mathrm{~K}$ & 0.276 \\
$392.37 \mathrm{~K} \leftrightarrow 401.31 \mathrm{~K}$ & 0.278 & $605.18 \mathrm{~K} \leftrightarrow 617.96 \mathrm{~K}$ & 0.273 \\
$401.31 \mathrm{~K} \leftrightarrow 410.42 \mathrm{~K}$ & 0.275 & $617.96 \mathrm{~K} \leftrightarrow 630.99 \mathrm{~K}$ & 0.270 \\
$410.42 \mathrm{~K} \leftrightarrow 419.68 \mathrm{~K}$ & 0.273 & $630.99 \mathrm{~K} \leftrightarrow 644.24 \mathrm{~K}$ & 0.267 \\
$419.68 \mathrm{~K} \leftrightarrow 429.10 \mathrm{~K}$ & 0.239 & $644.24 \mathrm{~K} \leftrightarrow 657.74 \mathrm{~K}$ & 0.261 \\
$429.10 \mathrm{~K} \leftrightarrow 438.71 \mathrm{~K}$ & 0.274 & $657.74 \mathrm{~K} \leftrightarrow 671.47 \mathrm{~K}$ & 0.258 \\
$438.71 \mathrm{~K} \leftrightarrow 448.48 \mathrm{~K}$ & 0.255 & $671.47 \mathrm{~K} \leftrightarrow 685.46 \mathrm{~K}$ & 0.272 \\
$448.48 \mathrm{~K} \leftrightarrow 458.42 \mathrm{~K}$ & 0.254 & $685.46 \mathrm{~K} \leftrightarrow 699.71 \mathrm{~K}$ & 0.251 \\
$458.42 \mathrm{~K} \leftrightarrow 468.48 \mathrm{~K}$ & 0.275 & $699.71 \mathrm{~K} \leftrightarrow 714.21 \mathrm{~K}$ & 0.266 \\
$468.48 \mathrm{~K} \leftrightarrow 478.79 \mathrm{~K}$ & 0.270 & $714.21 \mathrm{~K} \leftrightarrow 728.98 \mathrm{~K}$ & 0.265 \\
$478.79 \mathrm{~K} \leftrightarrow 489.28 \mathrm{~K}$ & 0.272 & $728.98 \mathrm{~K} \leftrightarrow 744.01 \mathrm{~K}$ & 0.284 \\
$489.28 \mathrm{~K} \leftrightarrow 499.96 \mathrm{~K}$ & 0.269 & $744.01 \mathrm{~K} \leftrightarrow 759.36 \mathrm{~K}$ & 0.280 \\
$499.96 \mathrm{~K} \leftrightarrow 510.78 \mathrm{~K}$ & 0.262 & $759.36 \mathrm{~K} \leftrightarrow 774.94 \mathrm{~K}$ & 0.273 \\
$510.78 \mathrm{~K} \leftrightarrow 521.85 \mathrm{~K}$ & 0.277 & $774.94 \mathrm{~K} \leftrightarrow 790.87 \mathrm{~K}$ & 0.279 \\
$521.85 \mathrm{~K} \leftrightarrow 533.12 \mathrm{~K}$ & 0.271 & $790.87 \mathrm{~K} \leftrightarrow 799.04 \mathrm{~K}$ & 0.250 \\
$533.12 \mathrm{~K} \leftrightarrow 544.59 \mathrm{~K}$ & 0.246 & $799.04 \mathrm{~K} \leftrightarrow 800.00 \mathrm{~K}$ & 0.270 \\
\hline
\end{tabular}


Table S4. Acceptance ratios of replicas corresponding to pairs of neighboring temperatures for $(S)$-3-quinuclidinol crystal.

\begin{tabular}{ll|lc}
\hline Pair of temperatures & Acceptance ratio & Pair of temperatures & Acceptance ratio \\
\hline $350.00 \mathrm{~K} \leftrightarrow 358.18 \mathrm{~K}$ & 0.270 & $544.59 \mathrm{~K} \leftrightarrow 556.28 \mathrm{~K}$ & 0.251 \\
$358.18 \mathrm{~K} \leftrightarrow 366.50 \mathrm{~K}$ & 0.255 & $556.28 \mathrm{~K} \leftrightarrow 568.18 \mathrm{~K}$ & 0.248 \\
$366.50 \mathrm{~K} \leftrightarrow 374.97 \mathrm{~K}$ & 0.266 & $568.18 \mathrm{~K} \leftrightarrow 580.29 \mathrm{~K}$ & 0.266 \\
$374.97 \mathrm{~K} \leftrightarrow 383.59 \mathrm{~K}$ & 0.263 & $580.29 \mathrm{~K} \leftrightarrow 592.62 \mathrm{~K}$ & 0.271 \\
$383.59 \mathrm{~K} \leftrightarrow 392.37 \mathrm{~K}$ & 0.271 & $592.62 \mathrm{~K} \leftrightarrow 605.18 \mathrm{~K}$ & 0.253 \\
$392.37 \mathrm{~K} \leftrightarrow 401.31 \mathrm{~K}$ & 0.253 & $605.18 \mathrm{~K} \leftrightarrow 617.96 \mathrm{~K}$ & 0.251 \\
$401.31 \mathrm{~K} \leftrightarrow 410.42 \mathrm{~K}$ & 0.259 & $617.96 \mathrm{~K} \leftrightarrow 630.99 \mathrm{~K}$ & 0.253 \\
$410.42 \mathrm{~K} \leftrightarrow 419.68 \mathrm{~K}$ & 0.261 & $630.99 \mathrm{~K} \leftrightarrow 644.24 \mathrm{~K}$ & 0.252 \\
$419.68 \mathrm{~K} \leftrightarrow 429.10 \mathrm{~K}$ & 0.257 & $644.24 \mathrm{~K} \leftrightarrow 657.74 \mathrm{~K}$ & 0.251 \\
$429.10 \mathrm{~K} \leftrightarrow 438.71 \mathrm{~K}$ & 0.253 & $657.74 \mathrm{~K} \leftrightarrow 671.47 \mathrm{~K}$ & 0.266 \\
$438.71 \mathrm{~K} \leftrightarrow 448.48 \mathrm{~K}$ & 0.252 & $671.47 \mathrm{~K} \leftrightarrow 685.46 \mathrm{~K}$ & 0.262 \\
$448.48 \mathrm{~K} \leftrightarrow 458.42 \mathrm{~K}$ & 0.251 & $685.46 \mathrm{~K} \leftrightarrow 699.71 \mathrm{~K}$ & 0.235 \\
$458.42 \mathrm{~K} \leftrightarrow 468.48 \mathrm{~K}$ & 0.256 & $699.71 \mathrm{~K} \leftrightarrow 714.21 \mathrm{~K}$ & 0.250 \\
$468.48 \mathrm{~K} \leftrightarrow 478.79 \mathrm{~K}$ & 0.257 & $714.21 \mathrm{~K} \leftrightarrow 728.98 \mathrm{~K}$ & 0.243 \\
$478.79 \mathrm{~K} \leftrightarrow 489.28 \mathrm{~K}$ & 0.260 & $728.98 \mathrm{~K} \leftrightarrow 744.01 \mathrm{~K}$ & 0.263 \\
$489.28 \mathrm{~K} \leftrightarrow 499.96 \mathrm{~K}$ & 0.250 & $744.01 \mathrm{~K} \leftrightarrow 759.36 \mathrm{~K}$ & 0.232 \\
$499.96 \mathrm{~K} \leftrightarrow 510.78 \mathrm{~K}$ & 0.262 & $759.36 \mathrm{~K} \leftrightarrow 774.94 \mathrm{~K}$ & 0.214 \\
$510.78 \mathrm{~K} \leftrightarrow 521.85 \mathrm{~K}$ & 0.259 & $774.94 \mathrm{~K} \leftrightarrow 790.87 \mathrm{~K}$ & 0.261 \\
$521.85 \mathrm{~K} \leftrightarrow 533.12 \mathrm{~K}$ & 0.263 & $790.87 \mathrm{~K} \leftrightarrow 799.04 \mathrm{~K}$ & 0.251 \\
$533.12 \mathrm{~K} \leftrightarrow 544.59 \mathrm{~K}$ & 0.246 & $799.04 \mathrm{~K} \leftrightarrow 800.00 \mathrm{~K}$ & 0.231 \\
\hline & & &
\end{tabular}

Table S5. The electric field strength $(E)$ and the characteristic switching time $\left(t_{0}\right)$ of $(R) /(S)$-3-quinuclidinol.

\begin{tabular}{ccccc}
\hline$E / \mathrm{V} \cdot \mathrm{nm}^{-1}$ & $(R)-t_{0} / \mathrm{ns}$ & $(S)-t_{0} / \mathrm{ns}$ & $(R)-\mathrm{n}$ & $(S)-\mathrm{n}$ \\
\hline 17 & 6.02 & 7.23 & 20.33 & 20.03 \\
18 & 3.24 & 3.05 & 20.01 & 19.56 \\
19 & 1.71 & 1.07 & 19.60 & 19.30 \\
20 & 0.95 & 0.30 & 14.47 & 14.38 \\
21 & 0.47 & 0.145 & 12.38 & 9.08 \\
22 & 0.28 & 0.065 & 6.05 & 8.03 \\
\hline
\end{tabular}




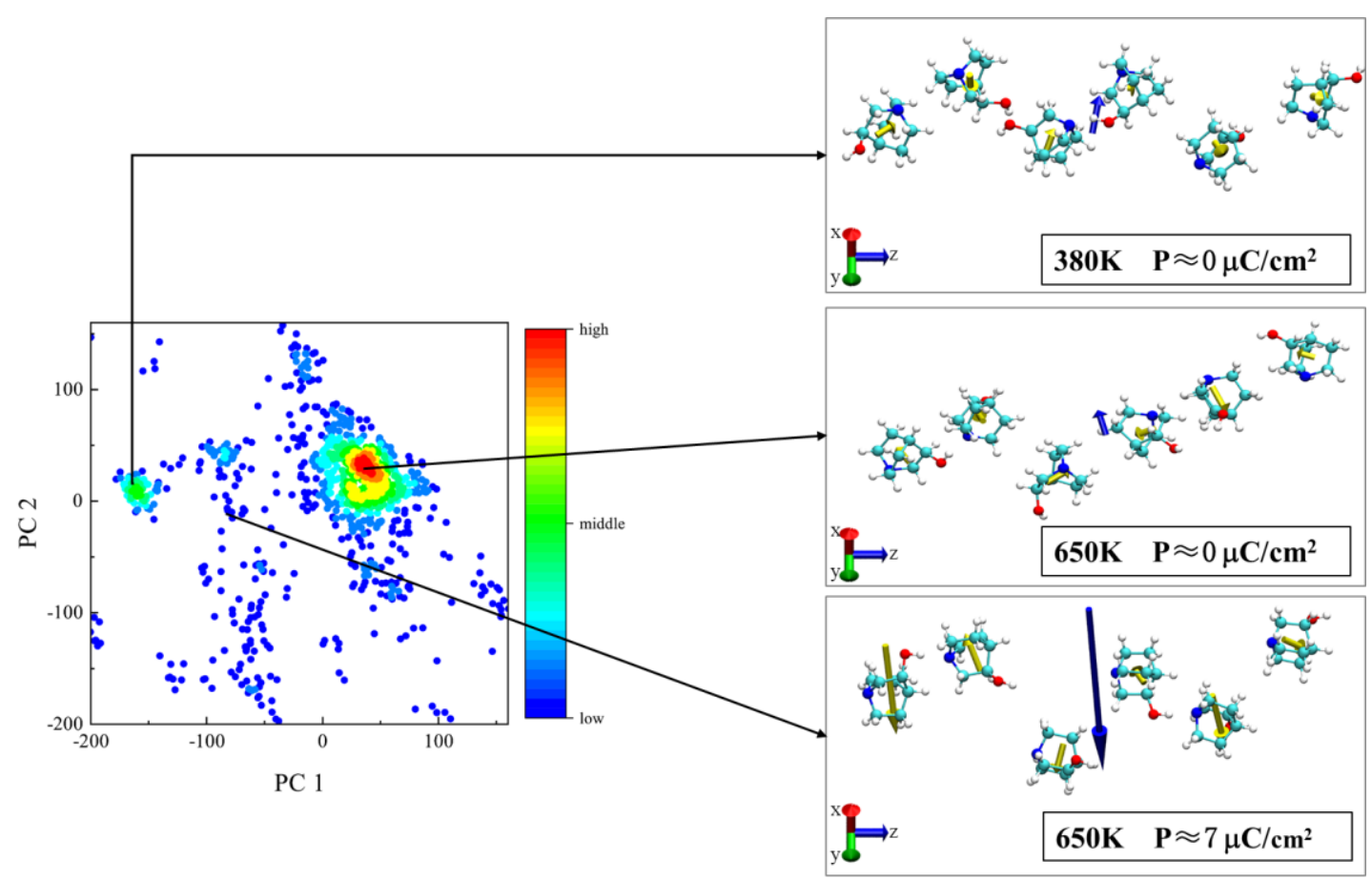

Figure S5: The dipole moments of three single molecule of $(R)$-3-quinuclidinol crystal are shown by yellow arrows, and the total dipole moments of the $(R)$-3-quinuclidinol crystal are shown by blue arrows.
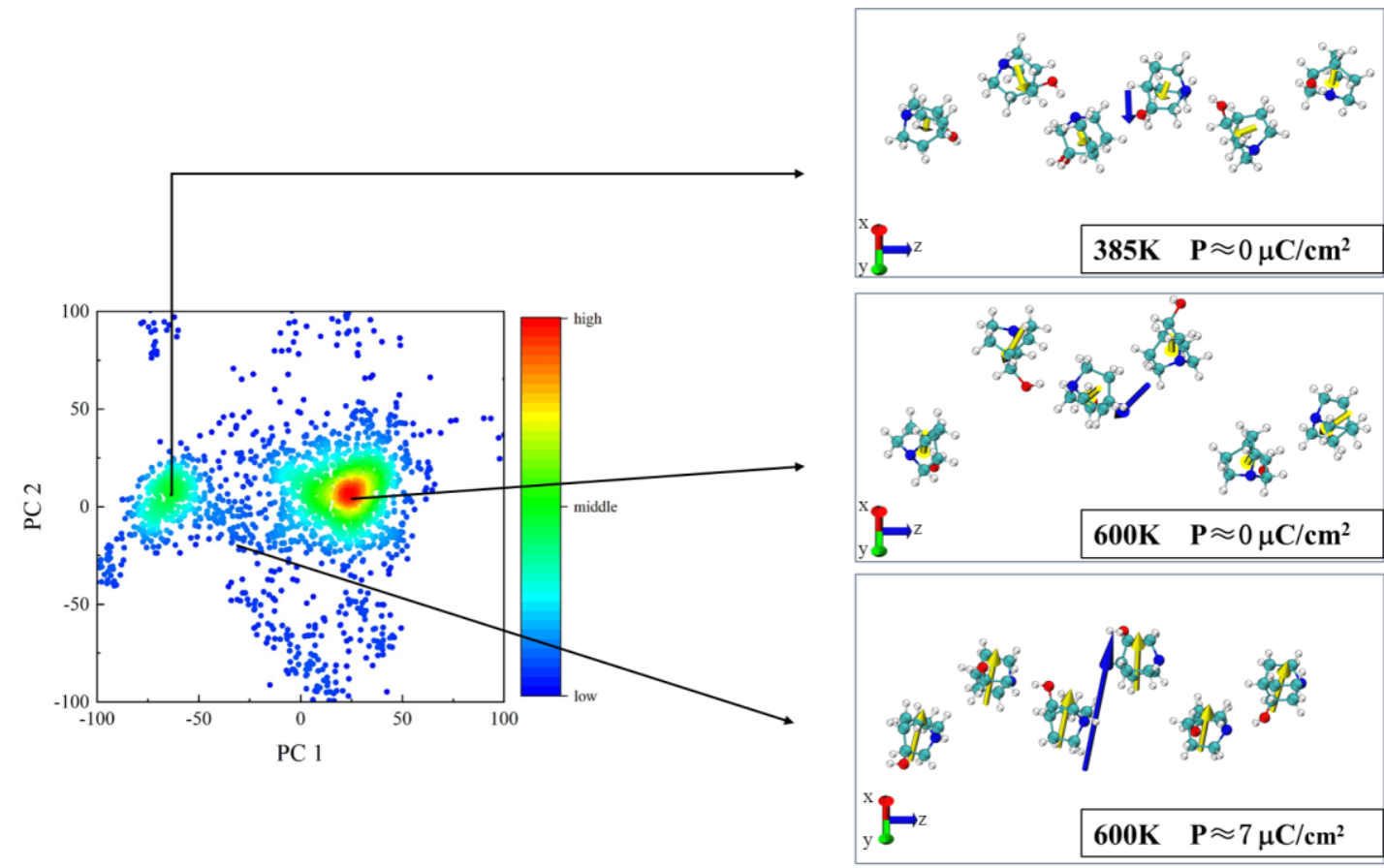

Figure S6: The dipole moments of each monomer molecule in the three conformations of $(S)$-3-quinuclidinol crystal are shown by yellow arrows, and the total dipole moments of the $(S)$-3-quinuclidinol crystal are shown by blue arrows. 

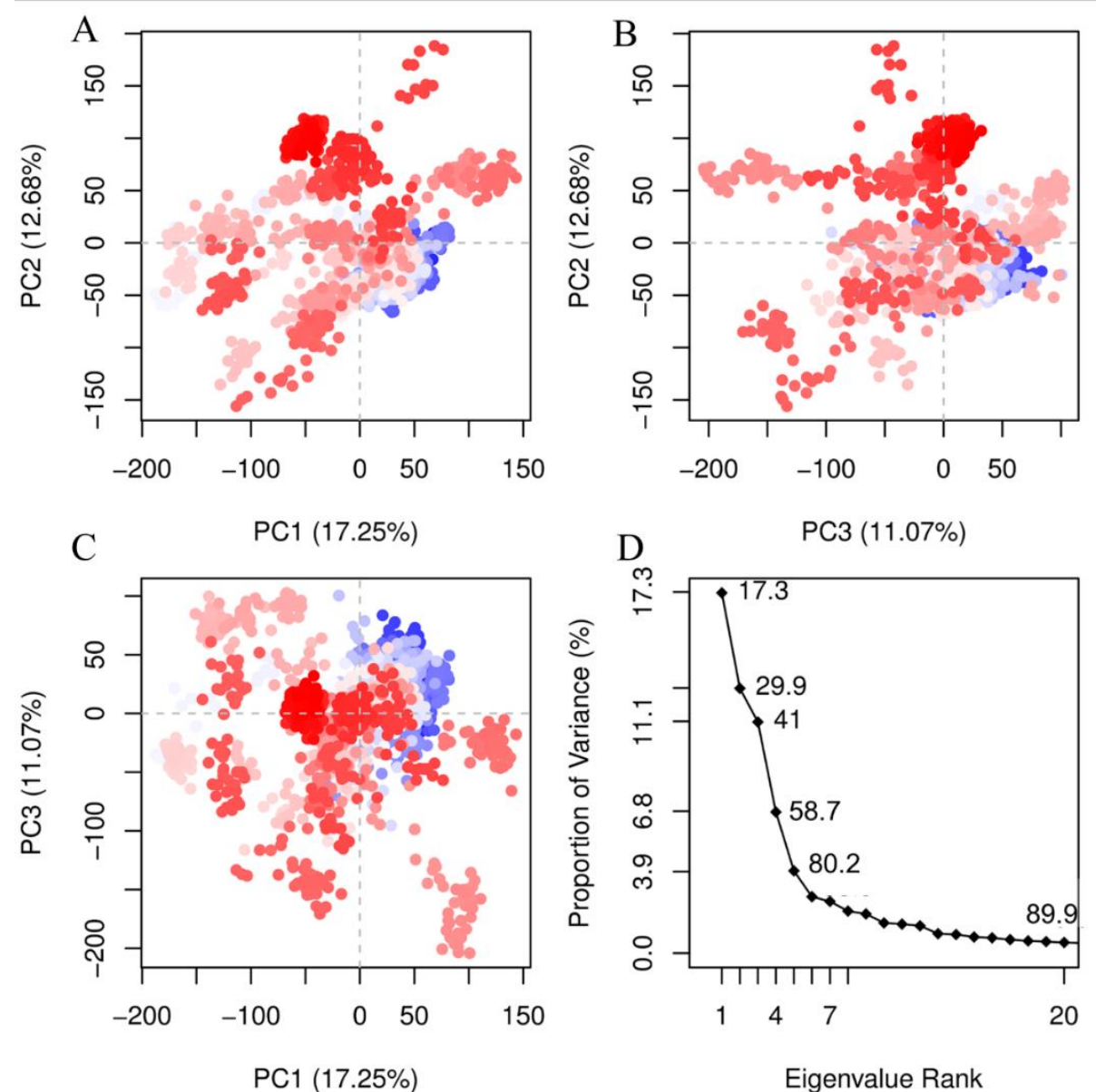

Figure S7: For replica-exchange dynamics simulation of $(R)$-3-quinuclidinol crystal, $\mathrm{A}$ is the distribution plot for the 1st and the 2nd PCs. B is the distribution plot for the 2nd and 3rd PCs. C is the distribution for the 1st and 3rd PCs. The D shows the first five PCs can cover over $80 \%$ kinetic information. 

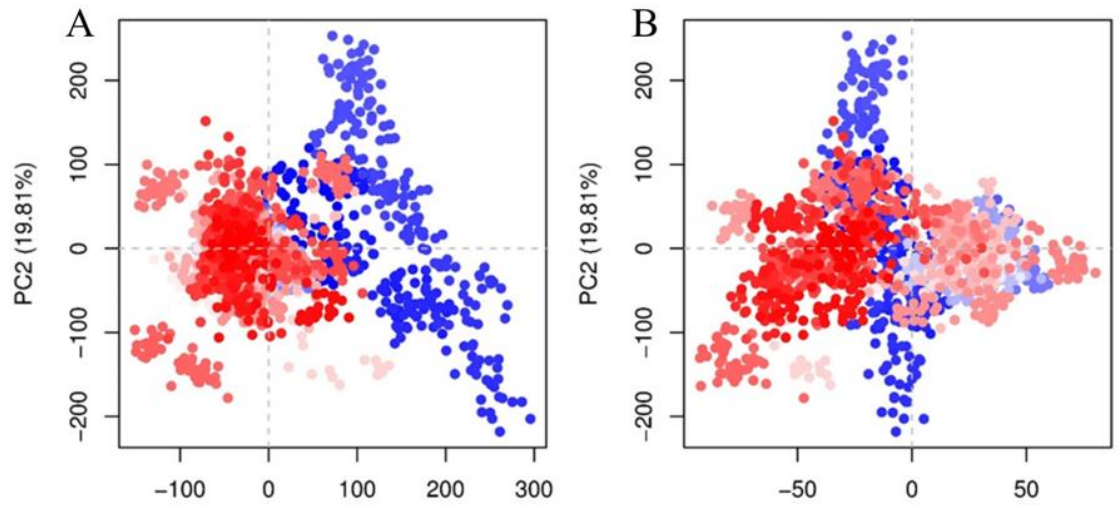

C

PC1 $(29.68 \%)$
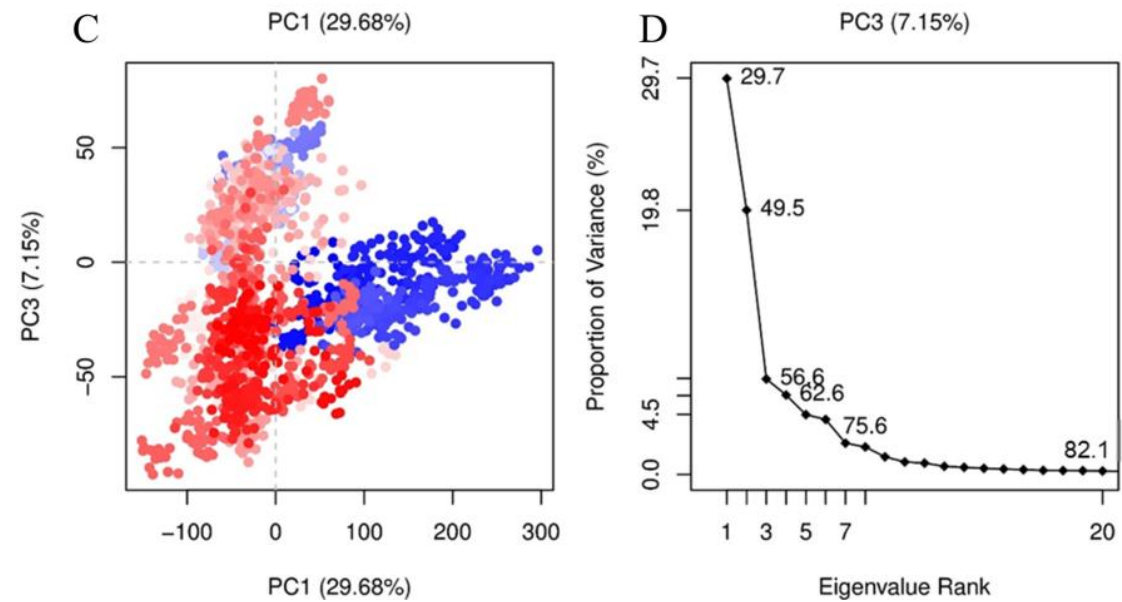

Figure S8: For replica-exchange dynamics simulation of (S)-3-quinuclidinol crystal, A is the distribution plot for the 1st and the 2nd PCs. B is the distribution plot for the 2nd and 3rd PCs. C is the distribution for the 1st and 3rd PCs. The D shows the first five PCs can cover over $70 \%$ kinetic information. 